\title{
Gauge theory in higher dimensions, II
}

\author{
Simon Donaldson and Ed Segal
}

\section{Contents}

1. Introduction 1

2. Basic set-up 2

2.1. Exceptional holonomy 2

2.2. Gauge theory and submanifolds 5

3. Taming forms 9

3.1. Dimension $8 \quad 9$

3.2. Dimension $7 \quad 10$

3.3. Dimension $6 \quad 12$

4. Gauge theory on tamed almost- $G_{2}$-manifolds with tubular ends 15

4.1. Compact 7-manifolds 15

4.2. 7-manifolds with tubular ends 17

4.3. Holomorphic bundles over moduli space 19

5. Finite-dimensional analogue 23

5.1. Morse-Novikov Theory 23

5.2. Discussion 28

6. Interaction between gauge theory and calibrated geometry 30

6.1. $G_{2}$-instantons and associative submanifolds 30

6.2. Implications 36

6.3. Codimension-3 theories and monopoles 38

$\begin{array}{ll}\text { References } & 40\end{array}$

\section{Introduction}

In this paper we follow up some of the ideas discussed in [6]. The theme of that article was the possibility of extending familiar constructions in gauge theory, associated to problems in low-dimensional topology, to higher dimensional situations, in the presence of an appropriate special geometric structure. The starting point for this was the "holomorphic Casson invariant", counting holomorphic bundles over a Calabi-Yau 3 -fold, analogous to the Casson invariant which counts flat connections over a

(C)2011 International Press 
differentiable 3-manifold. This was developed rigorously by Richard Thomas [36] in an algebro-geometric framework, and the ideas have been taken up by various authors [25]. From a differential-geometric standpoint one can make parallel discussions of two cases: one involving gauge theory and one involving submanifolds. There has been a considerable amount of work in a similar direction, involving ideas of Topological Quantum Field Theory [23], [31].

In the familiar gauge theory picture one views the Casson invariant as the Euler characteristic of the instanton Floer homology groups. Thus it is natural to hope for some analogous structure associated to a Calabi-Yau 3 -fold. This was discussed in a general way in [6] but the discussion there did not pin down exactly what structure one could expect. That is the goal of the present paper. In brief, we will argue that one should hope to find a holomorphic bundle over the moduli space of Calabi-Yau 3-folds, of rank equal to the holomorphic Casson invariant (sometimes called the DT invariant) defined by Thomas.

Just as in [6], many of the arguments here are tentative and speculative, since the fundamental analytical results that one would need to develop a theory properly are not yet in place. These have to do with the compactness of moduli spaces of solutions. While considerable progress has been made in this direction by Tian $[\mathbf{3 8}],[\mathbf{3 2}]$, a detailed theory - in either the gauge theory or submanifold setting - seems still to be fairly distant. The issues are similar to those involved in "counting" special Lagrangian submanifolds in Calabi-Yau manifolds, which have been considered by Joyce [20] but where, again, a final theory is still lacking.

The core of this article is Section 4, where we explain how to construct holomorphic bundles over Calabi-Yau moduli spaces, assuming favourable properties of a " $(6+1)$-dimensional" differential-geometric theory. The preceding Sections 2 and 3 develop background material, mostly fairly standard but introducing a point of view involving "taming forms". In Section 5 we explain how our construction matches up with standard algebraic topology, following the familiar Floer-theory philosophy. In Section 6 we go back to discuss the central, unresolved, compactness issues. We explain the relevance of recent work of Haydys which brings in a version of the "Fueter equation". This perhaps points the way to a unification of the gauge theory and calibrated geometry discussions and connections with the more algebrogeometric approach.

We are very grateful to Richard Thomas and Dominic Joyce for many discussions of this material. The paper has been substantially revised following comments of Joyce on an earlier draft. We are also grateful to Andriy Haydys for allowing us to present part of his forthcoming work.

\section{Basic set-up}

2.1. Exceptional holonomy. We will begin by recalling standard material on exceptional holonomy. Some references are $[\mathbf{1 9}]$, [30]. Start 
with the positive spin representation of $\operatorname{Spin}(8)$ on the 8-dimensional real vector space $S^{+}$. The basic fact is that this action maps onto the orthogonal group $S O\left(S^{+}\right)=S O(8)$. Likewise for the negative spin representation. This is the phenomenon of "triality": there are automorphisms of $\operatorname{Spin}(8)$ permuting the three representations $S^{+}, S^{-}, \mathbf{R}^{8}$. In particular the stabiliser in $\operatorname{Spin}(8)$ of a unit spinor in $S^{+}$is a copy of $\operatorname{Spin}(7) \subset \operatorname{Spin}(8)$, which maps to $\operatorname{Spin}(7) \subset S O(8)$. A Riemannian 8-manifold $X$ with a covariant constant unit spinor field has holonomy contained in $\operatorname{Spin}(7)$. In this situation we have a decomposition of the 2 -forms

$$
\Lambda^{2}=\Lambda_{21}^{2} \oplus \Lambda_{7}^{2}
$$

where $\Lambda_{21}^{2}$ corresponds to the Lie algebra of $\operatorname{Spin}(7)$, under the isomorphism $\Lambda^{2}=\mathfrak{s o}(8)$, and $\Lambda_{7}^{2}$ is the orthogonal complement. There is also a parallel 4 -form $\Omega$ which is equal to $\left(\sum \theta_{i}^{2}\right) / 7$ for any orthonormal basis $\theta_{i}$ of $\Lambda_{7}^{2}$. We can see this form in a useful explicit model. Suppose we have two copies $\mathbf{R}_{1}^{4}, \mathbf{R}_{2}^{4}$ of $\mathbf{R}^{4}$, each with spin structures. Then the positive spin space of $\mathbf{R}_{1}^{4} \oplus \mathbf{R}_{2}^{4}$ is the real part of

$$
\left(S^{+}\left(\mathbf{R}_{1}^{4}\right) \otimes S^{+}\left(\mathbf{R}_{2}^{4}\right)\right) \oplus\left(S^{-}\left(\mathbf{R}_{1}^{4}\right) \otimes S^{-}\left(\mathbf{R}_{2}^{4}\right)\right) .
$$

(Recall that the spin spaces in 4-dimensions are quaternionic and the complex tensor product of two quaternionic vector spaces has a natural real structure.) Fix an isomorphism $\Psi$ between $S^{+}\left(\mathbf{R}_{1}^{4}\right)$ and $S^{+}\left(\mathbf{R}_{2}^{4}\right)$. We can regard $\Psi$ as an element of the tensor product and we get a distinguished spinor in 8 dimensions. In other words we have a subgroup $H$ of $\operatorname{Spin}(7) \subset$ $S O(8)$, locally isomorphic to $S U(2) \times S U(2) \times S U(2)$, consisting of automorphims of $\mathbf{R}^{8}$ which preserve the decomposition $\mathbf{R}_{1}^{4} \oplus \mathbf{R}_{2}^{4}$ and $\Psi$. In this picture the 4 -form $\Omega$ corresponding to our distinguished spinor is

$$
d x_{1} d x_{2} d x_{3} d x_{4}+d y_{1} d y_{2} d y_{3} d y_{4}+\sum_{i=1}^{3} \omega_{i} \wedge \omega_{i}^{\prime} .
$$

Here $x_{i}, y_{i}$ are standard co-ordinates on the two copies of $\mathbf{R}^{4}, \omega_{i}$ is a standard orthonormal basis for $\Lambda^{+}\left(\mathbf{R}_{1}^{4}\right)$ and $\omega_{i}^{\prime}$ the basis of $\Lambda^{+}\left(\mathbf{R}_{2}^{4}\right)$ which corresponds to this under the isomorphism induced by $\Psi$. In fact this form $\Omega$ determines the spinor, so we could also define $\operatorname{Spin}(7) \subset G L(8, \mathbf{R})$ to be the stabiliser of this 4 -form. The $G L(8, \mathbf{R})$ orbit of $\Omega$ is a 43 -dimensional submanifold $\mathcal{A} \subset \Lambda^{4} \mathbf{R}^{8}$, which can be viewed as $G L(8, \mathbf{R}) / \operatorname{Spin}(7)$. On any 8-manifold we have a copy of $\mathcal{A}$ associated to each tangent space in the obvious way and a $\operatorname{Spin}(7)$ structure is equivalent to a closed 4 -form which takes values in this subset.

Now consider a unit spinor in $S^{+}$and a unit vector in $\mathbf{R}^{8}$. The stabiliser of the pair is the exceptional Lie group $G_{2}$, which can be regarded as a subgroup of $S O(7)$. This means that a Riemannian product $\mathbf{R} \times Y$ has holonomy contained in $\operatorname{Spin}(7)$ if and only if the holonomy of $Y$ is contained 
in $G_{2} \subset S O(7)$. On such a manifold $Y$ we have a decomposition of the 2forms

$$
\Lambda^{2}=\Lambda_{14}^{2} \oplus \Lambda_{7}^{2}
$$

where $\Lambda_{14}^{2}$ corresponds to the Lie algebra of $G_{2}$. We have a covariant constant 3 -form $\phi$ and 4 -form $\sigma$, such that on the cylinder

$$
\Omega=\phi d t+\sigma .
$$

We can define the concept of a non-compact Riemannian 8 manifold with holonomy in $\operatorname{Spin}(7)$ and a tubular end modelled on $(0, \infty) \times Y$, for a $G_{2}$-manifold $Y$. (That is, the metric differs from the product by an exponentially decaying term.) We can also consider a "neck-stretching" sequence of $\operatorname{Spin}(7)$-structures on a compact manifold that degenerate to a limit which is the disjoint union of two such non-compact manifolds. That is, the metrics contain regions which are almost isometric to long finite tubes $\left(-T_{i}, T_{i}\right) \times Y$, where $T_{i} \rightarrow \infty$.

Now we can repeat the discussion, starting in 7 dimensions. Considering $G_{2} \subset S O(7)$, the stabiliser of a unit vector is a copy of $S U(3) \subset S O(6)$. A Riemannian product $\mathbf{R} \times Z$ has a $G_{2}$-structure if and only if $Z$ is a CalabiYau 3-fold, with holonomy in $S U(3)$. Then we have a decomposition of the 2-forms

$$
\Lambda^{2}=\Lambda_{8}^{2} \oplus \Lambda_{7}^{2},
$$

where $\Lambda_{8}^{2}$ corresponds to the Lie algebra of $S U(3)$. There are covariant constant 3 -forms $\rho_{1}, \rho_{2}$ and a 2 -form $\omega$ such that on the cylinder

$$
\sigma=\rho_{2} d s+\omega^{2}, \quad \phi=\omega d s+\rho_{1} .
$$

Our notation here is that $s$ is the co-ordinate on $\mathbf{R}$. In fact either one of $\rho_{1}, \rho_{2}$ determines the other since

$$
\rho_{2}=-I \rho_{1},
$$

where $I$ is the parallel complex structure. From another point of view, the complex combination $\theta=\rho_{1}+i \rho_{2}$ is a holomorphic 3 -form on $Z$. The 2 -form $\omega$ lies in $\Lambda_{7}^{2}$, so we get a further decomposition

$$
\Lambda_{2}=\Lambda_{8}^{2} \oplus \Lambda_{6}^{2} \oplus\langle\omega\rangle .
$$

Again we may consider $G_{2}$ manifolds with tubular ends and neck-stretching sequences.

Here we stop, although we could repeat the process to consider CalabiYau 3-folds with tubular ends, etc. This kind of neck-stretching sequence, and the converse "gluing theory" for manifolds with tubular ends, is central in the work of Kovalev [21], and many interesting new examples have been found recently by Kovalev and Nordstrom [22]. 
2.2. Gauge theory and submanifolds. Next we review slightly less standard material on auxiliary differential geometric objects: submanifolds and connections. A fundamental reference for the first is the work of Harvey and Lawson [12]; a number of references for the second can be found in [6].

Start again in dimension 8. Take our model $\mathbf{R}_{1}^{4} \oplus \mathbf{R}_{2}^{4}$ above and consider the $\operatorname{Spin}(7)$-orbit of the 4-plane $\mathbf{R}_{1}^{4}$ in the Grassmannian of oriented 4-planes in $\mathbf{R}^{8}$. This is the set of Cayley 4-planes, and forms a 12-dimensional submanifold in the full Grassmannian (since the stabiliser of $\mathbf{R}_{1}^{4}$ in the 21-dimensional group $\operatorname{Spin}(7)$ is the 9 dimensional subgroup $H$ ). Another definition is that an oriented 4-plane $\Pi$ is Cayley if the restriction of $\Omega$ to $\Pi$ is the volume form. A third is that for any vector $v$ in $\mathbf{R}^{8}$

$$
\left.i_{v}(\Omega)\right|_{\Pi}=*_{\Pi}\left(\left.v^{\sharp}\right|_{\Pi}\right),
$$

where $*_{\Pi}$ is the $*$ operator on $\Pi$ induced by the metric and $v^{\sharp} \in\left(\mathbf{R}^{8}\right)^{*}$ is the dual of $v$, again defined by the metric.

Now in an 8-manifold with a $\operatorname{Spin}(7)$ structure we may consider Cayley submanifolds, whose tangent space at each point is Cayley. There are two fundamental properties of this condition:

- Property A The condition is an elliptic PDE. As a check on this, note that the condition is given locally by 4 equations, since the set of Cayley subspaces has codimension 4 in the full Grassmannian, while 4-dimensional submanifolds near to a given one can be represented by sections of the four-dimensional normal bundle. In fact the linearisation of the condition is given by a version of the Dirac operator acting on sections of the normal bundle [26].

- Property B The submanifold is calibrated: its volume is the topological invariant given by the integral of the closed form $\Omega$ and it is a minimal submanifold, minimizing volume in its homology class.

Next we move to gauge theory. We define a $\operatorname{Spin}(7)$-instanton to be a connection on a bundle $E$, with structure group $S U(l)$ say, whose curvature lies in $\Lambda_{21}^{2}$. Then we have, likewise:

- Property A The equation is elliptic, modulo gauge equivalences. The linearised theory can be formulated in terms of a bundle-valued version of the elliptic complex

$$
\Omega^{0} \stackrel{d}{\rightarrow} \Omega^{1} \stackrel{\pi_{7} \circ d}{\rightarrow} \Omega_{7}^{2}
$$

(Again, note the dimension check: $1-8+7=0$.)

- Property B The Yang-Mills energy is determined by the topology of the bundle. This comes from the algebraic fact that for $\alpha$ in $\Lambda_{21}^{2}$ we have

$$
\alpha \wedge \alpha \wedge \Omega=-|\alpha|^{2} \text { vol }
$$


Thus for a $\operatorname{Spin}(7)$-instanton:

$$
\int_{X^{8}}|F|^{2}=-\int_{X^{8}} \operatorname{Tr}\left(F^{2}\right) \wedge \Omega=8 \pi^{2} c_{2}(E) \cup \Omega[X] .
$$

It follows that Spin(7)-instantons are Yang-Mills connections. This theory has been developed in the thesis of C. Lewis [24].

Optimistically we could hope that these good properties would allow us to define invariants, modelled on the Gromov-Witten invariants of symplectic manifolds, in the submanifold case, and on the instanton invariants of 4-manifolds in the gauge theory case. In the simplest situation, where the relevant index or formal dimension is zero, one would seek to define a numerical invariant by counting solutions with appropriate signs. When the index is positive one could try to evaluate natural cohomology classes on the moduli space of solutions.

There is a third property - which one might call the Floer picture - of the equations, which becomes crucial when one considers manifolds with tubular ends and neck-stretching sequences. Consider a tube $I \times Y$, where $I \subset \mathbf{R}$ is an open interval, finite or infinite, and $Y$ is a $G_{2}$-manifold with a 4 -form $\sigma$. A connection $\mathbf{A}$ over $I \times Y$ yields a 1-parameter family $A_{t}$ of connections over $Y$.

Property $\mathbf{C}$ There is a locally-defined function $f$, on the space of connections over $Y$ modulo gauge equivalence, such that $\operatorname{Spin}(7)$ instantons correspond to gradient curves of $f$, and

$$
\frac{d}{d t} f\left(A_{t}\right)=-\left\|\frac{d A_{t}}{d t}\right\|^{2}=-\left\|F\left(A_{t}\right) \wedge \sigma\right\|^{2} .
$$

We know from analogous Floer-type theories that this is the essential property needed to control solutions over infinite tubes, and to obtain uniform control in neck-stretching sequences. The point is that one arrives in a situation where $f\left(A_{t}\right)$ is well-defined and the variation of $f$ over the interval is known, so the gradient property gives bounds on

$$
\int_{I}\left\|\frac{d A_{t}}{d t}\right\|^{2} d t, \quad \int_{I}\left\|F\left(A_{t}\right) \wedge \sigma\right\|^{2} d t
$$

To explain in a little more detail, we define a 1-form on the space of connections over $Y$ by mapping a tangent vector $\delta A$ to

$$
\int_{Y} \operatorname{Tr}(\delta A F) \wedge \sigma
$$

This 1-form arises, locally in the space of connections modulo gauge equivalence, as the derivative of a function which can be written schematically as

$$
f(A)=\int_{Y} C S(A) \wedge \sigma
$$


where $C S$ denotes the Chern-Simons form. Of course this is not really welldefined and a more precise definition is this. We choose a base point $A_{0}$ and for any connection $A$ we choose a connection $\mathbf{A}$ on a bundle over $[0,1] \times Y$ with boundary values $A_{0}$ and $A$. Then we define

$$
f(A)=\int_{[0,1] \times Y} \sigma \operatorname{Tr} F_{\mathbf{A}}^{2} .
$$

Just as in the usual Floer theory over 3-manifolds, this function is not globally well-defined, but the indeterminacy comes from the periods of $\sigma$ over $H_{4}(Y, \mathbf{Z})$. This indeterminacy is related the notion of an "adapted bundle" which we will discuss further in Section 4 . But in any case we have a welldefined closed 1-form on the space of connections over $Y$. The $\operatorname{Spin}(7)-$ instanton equation over the tube can be written as

$$
\frac{d A_{t}}{d t}=*\left(F\left(A_{t}\right) \wedge \sigma\right)
$$

which displays $A_{t}$ as an integral curve of the vector field dual to this 1-form. Likewise in the submanifold set-up, we define a 1-form on the space of 3-dimensional submanifolds of $Y$ by mapping a variation $v$-a vector field along a submanifold $P^{3} \subset Y^{7}$ - to

$$
\int_{P} i_{v}(\sigma)
$$

This 1-form is the derivative of a locally-defined function, determined by integrating $\sigma$ over cobordisms in $[0,1] \times Y$, and we have an analogue of Property $\mathrm{C}$ above.

Now we consider a connection on a bundle over $Y^{7}$ whose pull-back to the cylinder is a Spin(7)-instanton. Expressed directly over $Y$ this condition is just that

$$
F \wedge \sigma=0,
$$

and we call the solutions $G_{2}$-instantons. In the Floer picture, these are viewed as the zeros of the 1 -form on the space of connections.

A 3-dimensional submanifold $P \subset Y$ is called associative if $\mathbf{R} \times P$ is Cayley. In the picture above, such a submanifold is viewed as a zero of the 1 -form or critical point of the locally-defined function $f$. Associative submanifolds can be defined more directly by the condition that for any vector $v \in T Y$ the restriction to $P$ of the contraction $i_{v}(\sigma)$ vanishes. There is a basic algebraic model for the tangent space of $Y$ at a point of an associative submanifold like that which we saw in 8 dimensions. We consider a 3 -dimensional space $\mathbf{R}^{3}$ and a 2-dimensional complex vector space $V$ with symmetry group $S U(2)$. Then the tensor product of $V$ with the spin space $S=S\left(\mathbf{R}^{3}\right)$ has a real form $\mathbf{R}^{4}=(V \otimes S)_{\mathbf{R}}$ and we have a natural isomorphism $\Lambda_{+}^{2} \mathbf{R}^{4}=\mathbf{R}^{3}$. In other 
words, if $y_{1}, y_{2}, y_{3}$ are standard co-ordinates on $\mathbf{R}^{3}$ we have a corresponding basis $\omega_{1}, \omega_{2}, \omega_{3}$ for the self-dual 2 -forms on $\mathbf{R}^{4}$. Then we have a 3 -form

$$
\phi=\sum d y_{i} \omega_{i}+d y_{1} d y_{2} d y_{3}
$$

and a 4-form

$$
\sigma=\sum_{i, j, k \text { cyclic }} \omega_{i} d y_{j} d y_{k}+d x_{1} d x_{2} d x_{3} d x_{4} .
$$

This gives a standard model for the tangent space of a $G_{2}$-manifold at a point of an associative submanifold, with $y_{i}$ the co-ordinates on the submanifold.

A 4-dimensional submanifold $Q$ of $Y$ which is Cayley when regarded as a submanifold of $\mathbf{R} \times Y$ is called co-associative. The condition can be defined more directly by saying that the restriction of the 3 -form $\phi$ to $Q$ vanishes.

If we prefer, we can forget the 8-dimensional geometry and start directly in 7 dimensions, considering $G_{2}$ manifolds and three kinds of differential geometric objects: associative submanifolds, coassociative submanifolds and $G_{2}$-instantons. These three conditions enjoy the same crucial Properties A,B discussed above. The equations are elliptic when set up suitably, but this is now less straightforward. At first sight, the $G_{2}$-instanton equation imposes $7=\operatorname{dim} \Lambda_{7}^{2}$ conditions on the curvature of a connection, whereas we would expect to only impose $7-1$, taking account of gauge invariance. The explanation for this is that the curvature of any connection satisfies the Bianchi identity and the linearised problem can be formulated in terms of the elliptic complex

$$
0 \rightarrow \Omega^{0} \stackrel{d}{\rightarrow} \Omega^{1} \stackrel{\sigma \wedge d}{\rightarrow} \Omega^{6} \stackrel{d}{\rightarrow} \Omega^{7} \rightarrow 0
$$

Then the whole theory of local deformations of solutions to the $G_{2}$-instanton equations is closely analogous to the Taubes/Floer discussion of flat connections over 3-manifolds, with (21) taking the place of the de Rham complex over a 3-manifold. This theory has been developed in unpublished work of A. Tomatis and in the thesis of Henrique Sa Earp [29].

The associative condition is elliptic, with the linearisation given by a version of the Dirac operator acting on sections of the normal bundle. The co-associative condition is at first sight overdetermined, since it imposes 4 constraints on the sections of the 3 -dimensional normal bundle of a 4-dimensional submanifold of $Y^{7}$, but when set-up properly it becomes elliptic [26]. One way of doing this is to embed the discussion in that of Cayley submanifolds in the cylinder. Our third characterisation (10) of Cayley subspaces shows that if $Q$ is any Cayley submanifold in $\mathbf{R} \times Y$ then the $\mathbf{R}$-coordinate $t$ is a harmonic function on $Q$. Thus if $Q$ is compact it must be a co-associative submanifold in some "fixed time" slice.

In sum, we would optimistically hope first for a 7-dimensional theory, bearing on a compact $G_{2}$-manifold $Y$ and yielding invariants counting associative submanifolds, $G_{2}$-instantons and co-associative submanifolds. In the 
first two cases these could be viewed as generalising the Casson invariant (since in each case we are counting the zeros of a closed 1-form). But second we could hope for a $(7+1)$-dimensional theory, assigning Floer groups to $Y$ which should play the same role vis-à-vis the $\operatorname{Spin}(7)$ discussion, for manifolds with tubular ends and neck-stretching limits, as the ordinary Floer theory does to 4 -manifold invariants. Of course the second theory would be a refinement of the first, since we would view the Casson invariant as the Euler characteristic of the Floer groups.

Now we repeat the discussion, dropping dimension again. The $G_{2^{-}}$ instantons on $\mathbf{R} \times Z^{6}$ which are lifted up from $Z$ are connections with

$$
F \wedge \omega^{2}=0, \quad F \wedge \rho_{2}=0 .
$$

(The conditions $F \wedge \rho_{1}=0, F \wedge \rho_{2}=0$ are equivalent.) From the point of view of complex geometry, the second condition is that $F$ has type $(1,1)$, so the connection defines a holomorphic structure on the bundle. The other condition is the Hermitian Yang-Mills equation, and we know that the solutions correspond to "polystable" holomorphic bundles. Likewise a product $\mathbf{R} \times \Sigma$ is co-associative if and only if $\Sigma$ is a complex curve in $Z^{6}$. These geometric objects in $Z^{6}$ again have the same good properties: they are defined by elliptic equations and have topological volume/energy bounds. We will take this discussion of these $(6+1)$-dimensional theories further in Section 4. One can also discuss co-associative submanifolds in a similar framework [23], [31]. The corresponding objects in 6 dimensions are the special Lagrangian submanifolds, which can be described as the critical points of a locally-defined functional [37]. The co-associative submanifolds of a tube are the gradient curves of this functional.

\section{Taming forms}

3.1. Dimension 8. We will now take another point of view, beginning again in 8-dimensions. Our model is Gromov's notion of a symplectic form "taming" an almost-complex structure $[\mathbf{1 1}]$. Let $\Omega_{0}$ be the standard form on $\mathbf{R}^{8}$, as in equation (3). The convex hull of the set of negative squares $-\alpha \wedge \alpha$, for $\alpha$ in $\Lambda_{21}^{2}$ is a proper cone $K$ in $\Lambda^{4} \mathbf{R}^{8}$ so we have a dual cone of 4-forms $\Omega^{\prime}$ such that $\Omega^{\prime} \wedge \chi>0$ for non-zero $\chi \in K$. This is equivalent to saying that the quadratic form

$$
\alpha \mapsto-\alpha \wedge \alpha \wedge \Omega^{\prime}
$$

is positive definite on $\Lambda_{21}^{2}$. One can check that the subset of Cayley planes in $G r_{4}\left(\mathbf{R}^{8}\right) \subset \Lambda^{4} \mathbf{R}^{8}$ lies in the cone $K$, so such a 4 -form is also strictly positive on Cayley subspaces. Now suppose that $\Omega$ is any 4 -form on an 8 -manifold $X$ which lies in the preferred subspace $\mathcal{A}$ at each point. We could call this an "almost $\operatorname{Spin}(7)$ structure". Then Cayley submanifolds and $\operatorname{Spin}(7)$ instantons are defined and the equations are elliptic, just as before. However we lose the volume/energy identity for solutions. Suppose 
however we have a closed 4 -form $\Omega^{\prime}$ which, at each point, lies in the dual cone above. Then for any $\operatorname{Spin}(7)$-instanton we have a slightly weaker version of "Property B":

Property $B^{\prime}$

$$
\int_{X}|F|^{2} \leq-C \int_{X} \operatorname{Tr}\left(F^{2}\right) \wedge \Omega^{\prime}
$$

where the right hand side is a topological invariant. Here the constant $C$ depends only on $\Omega, \Omega^{\prime}$. Similarly, we get a volume bound for Cayley submanifolds. It seems reasonable to hope that the putative theory in the case of genuine $\operatorname{Spin}(7)$ structures extends to this more general situation; in any case we will assume this is so for the purposes of our discussion. We call such a pair $\left(\Omega, \Omega^{\prime}\right)$ a tamed almost-Spin $(7)$ structure on $X$. The advantage of this extension is that the notion is much more flexible. On the one hand, starting with a genuine $\operatorname{Spin}(7)$-structure, it gives us scope to deform the equations, for example to achieve transversality. This is essentially the use we will make of the idea in Section 4. On the other hand, tamed almost Spin(7)-structures should be much easier to construct than Spin(7)-structures, since the condition on $\Omega^{\prime}$ is an open condition on a closed 4 -form.

3.2. Dimension 7. We may consider dimension reductions of the theory, in this more general setting. Let $V$ be an oriented 7-dimensional vector space and let $G L_{+}(V)$ be the linear automorphisms of positive determinant. There is an open $G L_{+}(V)$-orbit $P_{3} \subset \Lambda^{3} V^{*}$ of "positive" forms, each of which has stabiliser isomorphic to the compact group $G_{2} \subset S L(V)$. Similarly there is an open orbit of positive 4-forms $P_{4} \subset \Lambda^{4} V^{*}$ and a $G L_{+}(V)$ equivariant diffeomorphism $*: P_{3} \rightarrow P_{4}$. We also denote the inverse map by $*$. The choice of notation is derived from the fact that each element $\phi \in P_{3}$ defines a natural Euclidean metric $g_{\phi}$ on $V$ and $* \phi$ is the usual Hodge dual defined by this metric. Likewise for any $\sigma \in P_{4}$.

Now consider an oriented 7-manifold $Y$. At each point $p \in Y$ we have open subsets $P_{3, p} \subset \Lambda^{3} T^{*} Y_{p}, P_{4, p} \subset \Lambda^{4} T^{*} Y_{p}$. We define an almost $G_{2}$ structure on $Y$ to be a 4-form $\sigma$ on $Y$ which lies in $P_{4, p}$ at each point $p$. Of course it is the same to start with a 3 -form $\phi$ which lies in $P_{3, p}$ at each point, and an almost $G_{2}$ structure defines a Riemannian metric on $Y$. If $\sigma$ is an almost $G_{2}$-structure then the form

$$
\Omega=\sigma+* \sigma d t
$$

yields an almost $\operatorname{Spin}(7)$ structure on the cylinder $\mathbf{R} \times Y$. Let $\phi^{\prime}, \sigma^{\prime}$ be respectively, a closed 3-form and 4-form on $Y$, so $\Omega^{\prime}=\sigma^{\prime}+\phi^{\prime} d t$ is closed 4-form on the cylinder. Then there is a certain open set of "taming pairs" $\left(\phi^{\prime}, \sigma^{\prime}\right)$ such that $\Omega^{\prime}$ is a taming form. The discussion of associative submanifolds in $Y$ proceeds just as before. They are defined by the condition that the restriction of $i_{v} \sigma$ vanishes, for each tangent vector $\sigma$. The condition is an 
elliptic equation of Dirac type and their volume is controlled by the integral of $\phi^{\prime}$ over $P$, a topological invariant.

The discussion of $G_{2}$-instantons is different in an important way. For a general $\sigma$ the equation $\sigma \wedge F=0$ for a connection is overdetermined and we do not expect any solutions. However we can consider connections over $\mathbf{R} \times Y$ with the property that the restriction to each slice $\{t\} \times Y$ lies in a fixed gauge equivalence class. This corresponds to a pair $(A, u)$ over $Y$ where $A$ is a connection on a bundle $E$ and $u$ is a section of the adjoint bundle $\mathfrak{g}_{E}$. Then we have a connection $A+u d t$ on the lifted bundle over $\mathbf{R} \times Y$. The $\operatorname{Spin}(7)$-instanton equation, expressed in 7-dimensions, becomes

$$
F \wedge \sigma=* d_{A} u
$$

where $*$ is the Hodge $*$-operator of the metric $g_{\sigma}$. This is an elliptic equation (modulo gauge equivalences) for the pair $(A, u)$. This set-up is similar to one considered in early work of Thomas: the advantage in our present situation is that, given a taming pair $\left(\sigma^{\prime}, \phi^{\prime}\right)$, we still get a topological bound like (23) on the Yang-Mills energy. (Although $\sigma^{\prime}$ does not appear explicitly we need to use it in deriving the inequality.)

Suppose however that $\sigma$ is a closed 4 -form on $Y$ (in which case we might prefer to restrict attention to taming pairs with $\sigma^{\prime}=\sigma$ ). Then the Bianchi identity implies that for any solution $(A, u)$ as above (over a compact manifold $Y$ ) we have $d_{A} u=0$. Thus in this case we do get a good theory of $G_{2}$-instantons, without the extra field $u$. The explanation is that when $\sigma$ is closed we have a locally defined "Chern-Simons functional" just as before, with critical points the $G_{2}$-instantons, and the linearised theory can be expressed in terms of an elliptic complex (21). Moreover we have exactly the same gradient curve description as before. To sum up

(1) If we restrict attention to tamed structures on 8-manifolds with tubular ends such that on each end the structure is defined by a closed 4 -form $\sigma$ on the cross section then we get a $(7+1)$ dimensional differential geometric theory with the good Properties $A, B^{\prime}, C$. So we have reasons to hope that some kind of Floer theory can be introduced into this more general and flexible, situation. The same remarks apply in the submanifold setting, for Cayley submanifolds with "associative limits".

(2) If, on the other hand, we are just interested in a compact 7-manifold $Y$ we can study solutions $(A, u)$ of equation (25) for any positive 4 -form $\sigma$, not necessarily closed, so long as there are taming forms. This equation has properties $A$ (with Fredholm index 0 ) and $B^{\prime}$, so we expect to define a numerical invariant. This can be regarded as counting the zeros of a vector field on the space of connections modulo gauge, but the vector field is not dual to a closed 1-form. Again, the same remarks apply to aasociative submanifolds. 
Remark We note in passing that, by contrast, we expect a good theory of coassociative submanifolds for almost $G_{2}$ structures where the 3-form $* \sigma$ is closed, rather than the 4 -form $\sigma$. The problem is that, for general $\sigma$ the equations are overdetermined so we lose Property A. The condition $d * \sigma=0$ is the integrability condition for the overdetermined system, much as the condition $d \sigma=0$ is the integrability condition for the $G_{2}$-instanton equations. One way of seeing this is to embed the discussion in that of Cayley submanifolds in the cylinder, as before.

3.3. Dimension 6. The discussion becomes considerably more confusing when we go down to 6 dimensions. We will need to have closed taming forms, comprising a 2-form $\omega^{\prime}$ a pair of 3 -forms $\rho_{1}^{\prime}, \rho_{2}^{\prime}$ and a 4 -form $\tau^{\prime}$. Thus on $\mathbf{R}^{2} \times Z$ our taming form will be

$$
\Omega^{\prime}=\omega^{\prime} d s d t+\rho_{1}^{\prime} d t+\rho_{2}^{\prime} d s+\tau^{\prime} .
$$

Then we will consider an $S U(3)$-structure on the tangent bundle. We recall that there is an open $G L(6, \mathbf{R})$-orbit of "positive" 3 -forms in $\Lambda^{3} \mathbf{R}^{6}$ each of which determines an almost-complex structure. Thus we can take our $S U(3)$-structure to be specified by a 3 -form $\rho_{1}$, which defines an almost complex structure and hence $\rho_{2}=-I \rho_{1}$ and a 2 -form $\omega$ which is a positive form of type $(1,1)$ with respect to this almost complex structure. The point to emphasise that, in this most general formulation, there are algebraic constraints on the forms $\omega, \rho_{1}$ but only open conditions on the taming forms. Then we get a wide variety of different extra conditions we can impose, intermediate between this most general formulation and the case of genuine Calabi-Yau structures.

Start with a 7 -manifold $Y$ with tubular ends and a tamed almost $G_{2^{-}}$ structure defined by a 4 -form $\sigma$, not necessarily closed. Then we want to study the equation (25), for pairs $(A, u)$ on $Y$. We need to have Property C: a gradient description on the ends. For this we consider a closed 3-form $\rho$ and the closed 4 -form $\tau$ on the cross-section $Z$ and the functional

$$
f(a, u)=\int_{Z} C S(a) \wedge \rho+\operatorname{Tr}(u F(a)) \wedge \tau,
$$

on pairs $(a, u)$ over $Z$. Suppose for the moment that we take an arbitrary Riemannian metric on $Z$ then we have a gradient equation

$$
\frac{d a}{d s}=*\left(F \wedge \rho+d_{a} u \wedge \tau\right), \quad \frac{d u}{d s}=*(F \wedge \tau)
$$

On the other hand, if we can write the 4 -form $\sigma$ as $\sigma=\rho_{2} d s+\omega^{2}$ the equation (25) becomes

$$
\omega^{2} \wedge \frac{d a}{d s}=\rho_{2} \wedge F+* d_{a} u, \quad \frac{d u}{d s}=*\left(F \wedge \omega^{2}\right)
$$

So we need to arrange that these equations are the same. First we should take $\tau=\omega^{2}$, that is we should suppose that $\omega^{2}$ is closed. Second we should 
suppose that $\rho=I \rho_{2}$ for the almost complex structure defined by $\rho_{2}$, and we should use the standard metric associated to $\omega$ and this almost-complex structure. Then the equations (26) and (27) are equivalent. But we can also use $\rho$ to define the same complex structure, and hence $\rho_{2}$. So if we start on $Z$ with a closed positive 3 -form $\rho$ and a 2 -form $\omega$ such that $\omega^{2}$ is closed and $\omega$ is positive of type $(1,1)$ with respect to the almost-complex structure defined by by $\rho$ we get a model for a tubular end on which equation (25) has a gradient description (Property $\mathrm{C}$ ). We can take $\rho, \tau$ as two of our taming forms $\rho_{1}^{\prime}, \tau^{\prime}$ and we also need another taming 3-form $\rho^{\prime}=\rho_{2}^{\prime}$ and a taming 2 -form $\omega^{\prime}$. We might want to suppose that in fact $\omega^{\prime}=\omega$. (The type $(1,1)$ condition is the same as saying that $\omega \wedge \rho=0$.) In this way we obtain a $(6+1)$-dimensional theory with Properties $A, B^{\prime}, C$. The stationary solutions over tubes correspond to pairs $(a, u)$ on $Z$ with

$$
F(a) \wedge \rho=d_{a} u \wedge \omega^{2} \quad F \wedge \omega^{2}=0 .
$$

The data we need over $Z$ consists of closed forms $\rho, \rho^{\prime}, \omega$ but the only identity (as opposed to an open condition) that we need to impose is $\rho \wedge \omega=0$. We expect that we then get Floer groups associated to $Z$, related to the invariants of almost $G_{2}$-structures in 7-dimensions.

If we want to study the 7-dimensional theory with $\sigma$ closed, which fits into the $(7+1)$ dimensional discussion, we are much more restricted. Then we need both $\rho$ and $I \rho$ to be closed 3-forms on $Z$, which can only occur if we have a genuine Calabi-Yau structure. In this case an integration-by-parts argument shows that for any solution of $(28), d_{a} u$ vanishes so we are back to the equations (22). In the opposite direction, if we consider the most general set up with no particular relation between the closed taming 3-forms and the $S U(3)$ structure, we can consider the system of equations for a triple $(a, u, v)$ where $a$ is a connection and $u, v$ are sections of $\mathfrak{g}_{E}$ :

$$
F \wedge \rho=d_{a} u \wedge \omega^{2}, \quad F \wedge(I \rho)=d_{a} v \wedge \omega^{2}, \quad F \wedge \omega^{2}=[u, v] \omega^{3} .
$$

We get a theory with Properties $A, B^{\prime}$ and we expect that counting solutions will generalise the holomorphic Casson invariant to this situation.

Much of the above has a good formal interpretation. The space of connections $\mathcal{A}$ on a bundle $E \rightarrow Z$ has a symplectic form

$$
\left\langle\delta_{1}, \delta_{2}\right\rangle=\int_{Z} \operatorname{Tr}\left(\delta_{1} \wedge \delta_{2}\right) \wedge \omega^{2} .
$$

The symplectic quotient $\mathcal{A} / / \mathcal{G}$ by the gauge group is given by solutions of the equation $F \wedge \omega^{2}=0$, modulo gauge equivalence. We have an induced symplectic form on $\mathcal{A} / / \mathcal{G}$. Any closed 3 -form $\tilde{\rho}$ yields a locally-defined function on the space $\mathcal{A} / \mathcal{G}$

$$
f_{\tilde{\rho}}(a)=\int_{Z} C S(a) \wedge \tilde{\rho}
$$


The equation $F \wedge \tilde{\rho}=d_{a} u \wedge \omega^{2}$ is the condition defining a critical point of $f_{\tilde{\rho}}$, restricted to the symplectic quotient $\mathcal{A} / / \mathcal{G} \subset \mathcal{A} / \mathcal{G}$. The evolution equation

$$
\frac{d a}{d t} \wedge \omega^{2}=F \wedge \tilde{\rho}
$$

defines the Hamiltonian flow associated to the function $f_{\tilde{\rho}}$ on $\mathcal{A} / / \mathcal{G}$. The difficulty in combining the $(7+1)$ and $(6+1)$-dimensional theories is that we want to find another 3 -form $\rho$ such that we get the same flow as the gradient flow of $f_{\rho}$, and this seems to essentially restrict us to the CalabiYau case. However this restriction may not be fundamental. We expect that the $(7+1)$ dimensional theory should be related, from the point of view of the 6-manifold $Z$, to a "Fukaya category" of Lagrangian submanifolds of $\mathcal{A} / / \mathcal{G}$ which would be, formally, something defined just by the symplectic structure of $\mathcal{A} / / \mathcal{G}$.

As usual, there is a corresponding discussion in the submanifold case. The infinite dimensional space of symplectic surfaces $\Sigma \subset Z$ has a natural symplectic form, arising formally as a symplectic quotient [7]. Then we get locally-defined functions on this space by integrating closed 3-forms over 3-dimensional cobordisms.

There is a variant of this discussion which yields deformation of the Special Lagrangian equations in a Calabi-Yau 3-fold. To explain this we set up some notation. Suppose $L$ is a submanifold of a manifold $M$ and $\psi$ is a $p$-form on $M$. If the restriction of $\psi$ to $L$ vanishes then $\psi$ defines a section of $\Omega^{p-1}\left(L, N^{*}\right)$ where $N$ is the normal bundle $T M / T L$ of $L$ in $M$. We will denote this section by $\psi_{N}$. Now consider a closed 3 -form $\rho$ and a closed 4 -form $\tau$ on a 6 -manifold $Z$. There is a locally defined functional $f_{\tau}$ on the space of 3 -dimensional submanifolds of $Z$, defined by integrating $\tau$ over 4-dimensional cobordisms. Let $\mathcal{C}_{\rho}$ be the set of submanifolds $P^{3} \subset Z^{6}$ such that the restriction of $\rho$ to $P$ vanishes. We consider the critical points of $f_{\tau}$ restricted to $\mathcal{C}_{\rho}$. For any submanifold $P \in \mathcal{C}_{\rho}$ the restrictions $\left.\rho\right|_{P},\left.\tau\right|_{P}$ both vanish, the first by definition and the second for dimensional reasons, so we have well-defined bundle-valued forms $\rho_{N} \in \Omega^{2}\left(N^{*}\right), \tau_{N} \in \Omega^{3}\left(N^{*}\right)$. The Euler-Lagrange equation defining the critical points involves a function $f$ on $P$ (which appears as a "Lagrange multiplier") and takes the form

$$
\tau_{N}=d f \wedge \rho_{N} .
$$

Thus we have a system of equations for a pair $(P, f)$ :

$$
\left.\rho\right|_{P}=0, \quad \tau_{N}=d f \wedge \rho_{N},
$$

which are analogous to (28). (Of course we need to factor out the constant functions $f$.) When $Z$ is a genuine Calabi-Yau manifold and $\rho, \tau$ are the standard forms the solutions are special Lagrangian submanifolds, as in [37], with $f=0$. More generally if we write $\tau=\omega^{2}$, where $\omega$ is not necessarily closed we can identify these pairs with associative submanifolds in the tube $Z \times \mathbf{R}$. One would expect that, for generic choices, the solutions $(P, f)$ are 
isolated and this deformation could be seen as removing a degeneracy in the special Lagrangian equations (which forces the latter to have solutions in moduli spaces of various dimensions, given by the first Betti number of $P$ ).

\section{Gauge theory on tamed almost- $G_{2}$-manifolds with tubular ends}

4.1. Compact 7-manifolds. We will now make a slightly more detailed analysis of the $(6+1)$-dimensional theory. We will do this in the gauge theory setting, but a similar discussion applies for the submanifold case. We will also restrict attention to a case when the cross-sections of the ends are genuine Calabi-Yau manifolds.

Suppose that $Y$ is a compact 7 -manifold with an almost $G_{2}$-structure defined by a 4 -form $\sigma$. Let $A$ be any connection on a bundle $E \rightarrow Y$ and form the sequence of operators

$$
\Omega^{0}\left(\mathfrak{g}_{E}\right) \rightarrow \Omega^{1}\left(\mathfrak{g}_{E}\right) \rightarrow \Omega^{6}\left(\mathfrak{g}_{E}\right) \rightarrow \Omega^{7}\left(\mathfrak{g}_{E}\right),
$$

as in (21). This is not in general a complex but we can make a single operator out of it in the usual way. Use the metric to identify $\Omega^{p}$ with $\Omega^{7-p}$ so we have

$$
D_{A}: \Omega^{0} \oplus \Omega^{1} \rightarrow \Omega^{0} \oplus \Omega^{1} .
$$

The elliptic operator $D_{A}$ is self-adjoint if and only if $\sigma$ is closed, but in any case its symbol is self-adjoint, so the index is zero. When $\sigma$ is closed and $A$ is a solution of the $G_{2}$-instanton equation we get an elliptic complex of Euler characteristic zero and we call $A$ regular if the cohomology of this complex vanishes. This implies in particular that $A$ is isolated in the moduli space of $G_{2}$-instantons.

We will discuss briefly two more technical issues reducible conections and orientations. Suppose first that $Y$ is manifold with holonomy equal to $G_{2}$. Then we know [19] that the harmonic 2-forms all lie in the $\Lambda_{14}^{2}$ component in (6). This means that any complex line bundle $L$ over $Y$ admits a $G_{2}$-instanton connection, and in particular such a connection appears as a reducible solutions $A_{0}$ on the bundle $E=L \oplus L^{-1}$. The situation is in some respects similar to that for instantons over a 4-manifold with negative definite intersection form. The bundle $\mathfrak{g}_{E}$ splits as $\mathbf{R} \oplus L^{2}$ and, at $A_{0}$, the complex (30) splits into a corresponding sum, with the interesting part given by

$$
\Omega^{0}\left(L^{2}\right) \rightarrow \Omega^{1}\left(L^{2}\right) \rightarrow \Omega^{6}\left(L^{2}\right) \rightarrow \Omega^{7}\left(L^{2}\right) .
$$

If the cohomology $H^{1}\left(L^{2}, A_{0}\right)$ vanishes then $A_{0}$ is isolated from irreducible solutions, and this is true also in families of small deformations of the $G_{2}$-structure. Thus, in this case, the irreducible solutions will not affect the enumerative discussion, counting the irreducible solutions. Since the complex has Euler characteristic 0 we expect that generically, in a family of $G_{2}$-structures, the cohomology will vanish. However we also expect that 
it will be non-trivial on some lower dimensional subset. The crucial point however is that the complex structure on $L^{2}$ means that we are considering families of complex linear operators and for these the generic picture is that cohomology will appear in real codimension 2. In that case there will be no interaction between the reducible and irreducible solutions in generic 1-parameter families, which is what are relevant to our purpose. So, granted that a more detailed analysis is necessary we take this as an indication that we can ignore the potential complications from reducible solutions, and for simplicity we just ignore these reducibles in what follows. (Similar remarks apply to reductions $S(U(p) \times U(q)) \subset S U(l)$.)

Now it seems very reasonable to assume that for generic $\sigma$ all solutions are regular Even if this is not the case we could contemplate introducing further, more artificial, perturbations of the equations or ideas of "virtual cycles", but let us assume that perturbations of $\sigma$ suffice. Our basic goal, when the structure is tamed by some closed form $\phi$ is to define a number by counting the solutions with appropriate signs, and it is the issue of these signs which we take up next. As usual, we mimic the standard discussion in the Casson-Floer theory over 3-manifolds. We seek to define a "relative sign" $\epsilon\left(A, A^{\prime}\right) \in\{ \pm 1\}$ for pairs of solutions with the property that

$$
\epsilon\left(A, A^{\prime \prime}\right)=\epsilon\left(A, A^{\prime}\right) \epsilon\left(A^{\prime}, A^{\prime \prime}\right) .
$$

This gives a way to attach signs to each solution, up to a single overall sign ambiguity. We define $\epsilon\left(A, A^{\prime}\right)$ using the spectral flow of a family of operators $D_{A_{t}}$, where $A_{t}$ is a path from $A$ to $A^{\prime}$. Given a path, this spectral flow yields an integer and we set $\epsilon$ to be 1 or -1 as the spectral flow is even or odd. Then the essential thing is to check that this independent of the path, which is the same as saying that the spectral flow around a closed loop (in the space of connections modulo gauge equivalence) is even. Such a loop yields a connection on a bundle $\mathbf{E}$ over $X=Y \times S^{1}$ and the spectral flow appears as the index of an elliptic operator over $X$. In fact this operator is just the operator apearing in the linearisation of the Spin(7)-instanton equation and can be identified simply as the Dirac operator over $X$, coupled to the bundle $\mathfrak{g}_{\mathbf{E}}$. Here the spin spaces in 8 dimensions are regarded as 8-dimensional real vector bundles. So this question of "orientability" in our 7-dimensional setup reduces to an algebro-topological question of showing that the index of this such a coupled Dirac operator over $X$ is even. (More generally, if the index of all such bundles is divisible by some integer $k$ then we expect the putative Floer theory associated to $Y$ to be $\mathbf{Z} / k$-graded.)

We can use the Atiyah-Singer index theorem to express this question in terms of characteristic classes. (An explicit formula is given by Lewis in [24].) But we can also give an argument which avoids detailed calculation. Since it is odd-dimensional, the manifold $Y$ has a nowhere vanishing vector field. This gives a reduction of the structure group of $Y$ to $S U(3) \subset G_{2}$, and hence of $X$ to $S U(3) \subset G_{2} \subset \operatorname{Spin}(7) \subset S O(8)$. With this reduction the spin bundles of $X$ acquire complex structures (corresponding to $V \oplus \mathbf{C}, V^{*} \oplus \mathbf{C}$ 
where $V$ is the fundamental representation of $S U(3)$. Of course we are not saying that these reductions are compatible with the differential geometric structure, but they are at the level of the symbol of the Dirac operator. Thus we can deform the coupled Dirac operator over $X$ to a complex linear operator, and hence the (real) index is even. However we will not try to develop the theory of orientations any further here.

We move on from this brief outline, which indicates how-modulo questions of compactness - we should define an integer counting the $G_{2^{-}}$ instantons on a bundle $E$ over $Y$. For each homology class $b \in H_{3}(Y)$ we consider $S U(l)$ bundles $E$ with $c_{2}(E)$ the Poincaré dual of $b$ and (for simplicity) with $c_{3}(E)=0$. There are at most a finite number of different topological types and we define an integer $n_{b}$ by summing the counts above over all such bundles. We expect this to be a deformation invariant, with respect to perturbations of $\sigma$. The energy bound implies that $n_{b}=0$ if $[\phi](b)<0$, since the moduli space is then empty. In fact $n_{b}$ vanishes for $b$ outside some proper cone in the half-space $\{b: \phi(b)>0\}$. We package these numbers into a formal series

$$
f_{Y}(\psi)=\sum n_{b} \exp (-\langle b, \psi\rangle)
$$

and our further hypothesis is that this converges to yield a holomorphic function of variable $\psi$ in an open subset of $H^{3}(Y, \mathbf{C})$ containing the points $r[\phi]$, for large enough $r$.

4.2. 7-manifolds with tubular ends. Now we discuss dimension 6 . If we have a Hermitian-Yang-Mills connection on a holomorphic bundle over a Calabi-Yau manifold $Z^{6}$, as in the previous section, the linearisation of the equations (28) for pairs $(a, u)$ yields an elliptic deformation complex of Euler characteristic zero. We call a solution regular if the cohomology vanishes, and this is just the same as saying the sheaf cohomology $H^{*}\left(Z, \mathfrak{g}_{E} \otimes \mathbf{C}\right)$ vanishes. We will assume that all solutions over $Z$ are regular. This is a very restrictive assumption, and we will return to discuss it further below.

Now suppose that $Y$ is a non-compact manifold with tubular ends each with a Calabi-Yau cross section, and that we have an almost $G_{2}$-structure defined by a closed form $\sigma$, compatible with the product structure on the ends, up to an exponentially decaying term. If we fix a solution over each end then we have the notions of an "adapted bundle" and "adapted connection" over $Y$, just as in the usual $3+1$ dimensional theory described in [8]. These are, respectively, a bundle over $Y$ with a fixed isomorphism with the pullbacks of the chosen bundles over the ends, and a connection over $Y$ which agrees with the model determined by the 6 -dimensional solution over each end, up to an exponentially decaying term.

Now fix attention on any adapted connection $A$ over $Y$ and form the differential operator $D_{A}$ as above. This is formally self-adjoint, just as before. Over an end, assuming that the connection is actually equal to the model, 
we can write $D_{A}$ as

$$
D_{A}=L \frac{d}{d t}+Q
$$

where $L$ is a skew-adjoint algebraic operator and $Q$ is a first-order selfadjoint operator, both over $Z$. Under our hypothesis that $Z$ is Calabi-Yau the composite $L^{-1} Q$ is a self-adjoint first order operator over $Y$ and the analysis of $D_{A}$ follows very closely that in the three-dimensional theory, as described in [8] for example. We deduce that $D_{A}: L_{1}^{2} \rightarrow L^{2}$ is Fredholm provided that the operator $Q$ over $Z$ does not have a zero eigenvalue. In turn, this is the same as the hypothesis that the solution over $Z$ is regular (since the operator $Q$ is essentially the same as that arising in the deformation theory over $Z$ ).

In the usual Floer theory we go on to consider the Fredholm index, which yields an invariant of an adapted bundle. The distinctive feature of the theory we are considering here is that the analogous index always vanishes. This is just a consequence of the fact, from the general theory, that the cokernel of $D_{A}$ is represented by the kernel of the formal adjoint, which is the same as $D_{A}$. The consequence is that, under our restrictive hypotheses, the study of $G_{2}$-instantons on a fixed adapted bundle over $Y$ behaves just like the compact case. We define regular solutions in just the same way, we expect that for generic $\sigma$ all solutions are regular and that a count of solutions yields a deformation invariant (with respect to compactly supported variations in $\sigma$ ) of the adapted bundle.

Now we discuss "neck stretching sequences" and gluing constructions. For simplicity consider a pair of manifolds $Y_{1}, Y_{2}$ each with one end having the same model $Z$ and appearing as the limit of a sequence of structures $\sigma_{T}$ on a compact manifold $Y$. (But note that when interchanging $Y_{1}, Y_{2}$ we have to change the sign of the 3 -form on $Z$.) Given regular $G_{2}$-instantons on adapted bundles over $Y_{1}, Y_{2}$ we wish to construct a glued solution over $\left(Y, \sigma_{T}\right)$ for large $T$. The proof follows the familiar Floer theory case closely, with one extra step. For large $T$ we construct an approximate solution $A_{0}$ with all norms of the error term $\sigma_{T} \wedge F\left(A_{0}\right)$ bounded by decaying exponential functions of $T$. Now we seek to solve the equation

$$
\sigma_{T} \wedge F\left(A_{0}+\alpha\right)=* d_{A_{0}+\alpha} u
$$

over $Y_{T}$, for a bundle valued 1-form $\alpha$ and 0 -form $u$. We also impose the gauge fixing condition $d_{A_{0}}^{*} \alpha=0$. Schematically, these equations can be written as

$$
D_{A_{0}} s=s * s+\sigma_{T} \wedge F\left(A_{0}\right)
$$

where $s$ is the pair $(\alpha, u)$, the notation $s * s$ denotes a quadratic algebraic term and $D_{A_{0}}$ is our basic elliptic operator. Mimicking the arguments in the $(3+1)$-dimensional case, we get a bound on the operator norm of the inverse of $D_{A_{0}}$ which is independent of $T$. Then the inverse function theorem shows that when $T$ is large (so $\sigma_{T} \wedge F\left(A_{0}\right)$ is small) there is a small solution $s$. 
Now the final extra step is to observe that, since $\sigma_{T}$ is closed, the Bianchi identity implies that in fact $d_{A} u=0$, as we have seen before. Hence $A$ is the desired $G_{2}$-instanton.

Still following the familiar pattern, we hypothesise that when $T$ is large, all $G_{2}$-instantons over $\left(Y, \sigma_{T}\right)$ arise by this gluing construction. Given a class $b \in H_{3}(Y)$ we let $c \in H_{2}(Z)$ be the image of $b$ under the boundary map of the Mayer-Vietoris sequence of $Y=Y_{1} \cup Y_{2}$. This is the same as the Poincare dual on $Z$ of the restriction of the Poincare dual, $P D_{Y}(b)$, of $b$ on $Y$. We use the symbol $\mathcal{S}$ to denote a solution over $Z$ on a bundle with Chern class the Poincaré dual of $c$. Then our hypothesis gives a gluing formula of the shape

$$
n_{b}=\sum_{\mathcal{S}} \sum_{E_{1}, E_{2}} n\left(E_{1}\right) n\left(E_{2}\right),
$$

where in the inner sum $E_{1}, E_{2}$ run over adapted bundles with common limit $\mathcal{S}$ and such that the glued bundle over $Y$ has $c_{2}=P . D .(b)$. Of course we have similar formulae when we glue manifolds with more than one end.

It is important to emphasise that if we work with almost $G_{2}$ structures where $\sigma$ is not closed we would get a different, richer theory, more like Floer theory over 3-manifolds and 4-manifolds with tubular ends. The operator $D_{A}$ is not self-adjoint and the index gives a non-trivial invariant of adapted bundles. Then we would get moduli spaces of different dimensions, depending on the index. However we will not pursue this further here.

4.3. Holomorphic bundles over moduli space. We have now reached the main question we wish to address in this paper. The gluing formula (33) is, in the general Floer theory framework, at the "chain level". As we vary the Calabi-Yau structure on $Z$ the solutions $\mathcal{S}$ vary and we do not have a canonical way to identify them. Further, even if choose such an identification locally the individual numbers $n_{E_{1}}, n_{E_{2}}$ will change. So we seek a more invariant way of expressing the formula much as, in the ordinary Floer theory, we pass from the "chains" to the Floer homology groups.

Let us for simplicity suppose that the inclusion of $Z$ in $Y$ induces an injection on $H_{3}$ and fix a coset $\left[b_{0}\right]$ in $H_{3}(Y) / H_{3}(Z)$. We restrict attention to classes $b$ in this coset, which have the same image $c$ in $H_{2}(Z)$. Now we consider a series like (32)

$$
g_{Y}(\psi)=\sum_{b \in H_{3}(Z)} n_{b+b_{0}} \exp (-\langle b, \psi\rangle)
$$

where now $\psi$ lies in an suitable open set in $H^{3}(Z, \mathbf{C})$, containing the points $r[\theta]$ for large enough $r$.

Fix the class $[\omega] \in H^{2}(Z)$ and consider the moduli space $\mathcal{M}$ of pairs $(I, \theta)$ where $I$ is a complex structure on $Z$ which admits Kähler metrics in the class $[\omega]$ and $\theta$ is a nowhere-zero holomorphic 3-form. By the Torelli theorem for 
Calabi-Yau manifolds this is a quotient $\tilde{\mathcal{M}} / \Gamma$ where $\Gamma$ is the symplectic mapping class group and $\tilde{\mathcal{M}}$ is immersed in $H^{3}(Z, \mathbf{C})$. We have an obvious $\mathbf{C}^{*}$-action on $\mathcal{M}$, multiplying the holomorphic form by a constant. We can also define a "norm" function by

$$
\|(I, \theta)\|^{2}=\int_{Z} \theta \wedge \bar{\theta}
$$

and for $R>0$ we write $\mathcal{M}_{R}$ for the points in $\mathcal{M}$ of norm greater than $R$. We suppose $R$ is some fixed, suitably large, number. Then the restriction of the holomorphic function $g_{Y}$ defines a holomorphic function on an open set in $\mathcal{M}_{R}$ containing the ray $\left.l_{\theta}=\{I, r \theta): r \geq 1\right\}$. We denote this restriction also by $g_{Y}$.

Now we make the assumption that for generic points in the moduli space $\mathcal{M}$ all solutions $\mathcal{S}$ are regular.

TENTATIVE PREDICTION 1. Under this assumption:

- There should be a holomorphic vector bundle $\mathcal{E} \rightarrow \mathcal{M}_{R}$, associated to the class $c \in H_{2}(Z)$, and a canonical isomorphism $(-1)^{*} \mathcal{E} \cong \mathcal{E}^{*}$.

- There should be an invariant $g_{Y_{1}}$ which is a holomorphic section of $\mathcal{E}$ over a neighbourhood of the ray $l_{\theta}$. Likewise there should be an invariant $g_{Y_{2}}$ which is a holomorphic section of $(-1)^{*} \mathcal{E}$ over a neighbourhood of $l_{\theta}$.

- The function $g_{Y}$ should be the dual pairing $\left\langle g_{Y_{1}}, g_{Y_{2}}\right\rangle$, formed using the isomorphism above

In other words, in this situation, the structure analogous to the Floer homology of a 3 -manifold is the holomorphic vector bundle $\mathcal{E}$ over the moduli space, and its sheaf of holomorphic sections.

\section{Remarks}

1. The mapping class group may not act freely on $\tilde{\mathcal{M}}$, in which case $\mathcal{M}$ is an orbifold. Then we more should strictly work with an "orbibundle" over $\mathcal{M}$.

2. When we restrict the function $g_{Y}$ from an open set in $H^{3}(Z, \mathbf{C})$ to $\tilde{\mathcal{M}}$ we could lose information. But our bundles actually extend over a thickening of $\mathcal{M}$ obtained from a quotient of such an open set and if formulated this way we get a gluing formula for the original function $g_{Y}$.

Now we will explain the construction of the bundle. Suppose that $\theta, \theta^{\prime}$ are two nearby Calabi-Yau structures on $Z$ and that they are generic in the sense above, so all solutions are regular. We have a collection of critical points $\{\mathcal{S}\}$ associated to $\theta$ (with the fixed class $c$ ) and another collection $\mathcal{S}^{\prime}$ associated to $\theta^{\prime}$. Let $V, V^{\prime}$ be the complex vector space with basis elements $\langle\mathcal{S}\rangle$ and $\left\langle\mathcal{S}^{\prime}\right\rangle$ respectively. Write $\langle\mathcal{S}\rangle^{*}$ for elements of the dual basis of $V^{*}$. We can choose a tamed almost $G_{2}$-structure on the topological cylinder $\mathbf{R} \times Z$ 
which is asymptotic to that defined by $\theta$ at $-\infty$ and by $\theta^{\prime}$ at $+\infty$. Fix, for the moment, closed 3-forms $\psi$ representing cohomology classes $\psi \in H^{3}(Z)$ and extend these to closed 3 -forms $\bar{\psi}$ on the topological cylinder, compatible with the product structure on the ends. We choose the taming form $\phi$ as the representative of $\boldsymbol{R e}(\theta)$. Then for each adapted bundle $E$ over the cylinder we have the following

1. Asymptotic limits $\mathcal{S}(E) \in\{\mathcal{S}\}, \mathcal{S}^{\prime}(E) \in\left\{\mathcal{S}^{\prime}\right\}$, at $t= \pm \infty$ respectively.

2. A real number

$$
\underline{\psi}(E)=-\frac{1}{8 \pi^{2}} \int_{Z \times \mathbf{R}} \bar{\psi} \operatorname{Tr}\left(F_{A}\right)^{2},
$$

where $A$ is any adapted connection. This is independent of the choice of connection and of the representative $\bar{\psi}$, given a choice of $\psi$.

3. An integer $n(E)$ counting the number of $G_{2}$-instantons.

Now we define a holomorphic function with values in $\operatorname{Hom}\left(V, V^{\prime}\right)$

$$
G_{\theta, \theta^{\prime}}(\psi)=\sum_{E} n(E) \exp (-\underline{\psi}(E))\langle\mathcal{S}(E)\rangle^{*} \otimes\left\langle\mathcal{S}^{\prime}(E)\right\rangle .
$$

This will be defined in a neighbourhood of the ray $l_{\theta}$. If we change the choice of representive $\underline{\psi}$ of a class $\psi$ to $\underline{\psi}+d \lambda$ we change the numbers $\underline{\psi}(E)$ to

$$
\underline{\psi}(E)+\int_{Z}\left(\operatorname{Tr}\left(F_{\mathcal{S}(E)}^{2}\right)-\operatorname{Tr}\left(F_{\mathcal{S}^{\prime}(E)}^{2}\right) \lambda .\right.
$$

This has the effect of changing the linear map $G_{\theta, \theta^{\prime}}(\psi)$ to

$$
\Lambda_{\theta}(\psi) G_{\theta, \theta^{\prime}}(\psi) \Lambda_{\theta^{\prime}}(\psi)^{-1},
$$

where in our bases $\Lambda_{\theta}, \Lambda_{\theta^{\prime}}$ are diagonal matrices with entries given by the exponentials of

$$
\int_{Z} \operatorname{Tr} F_{\mathcal{S}}^{2} \lambda, \quad \int_{Z} \operatorname{Tr} F_{\mathcal{S}^{\prime}}^{2} \lambda
$$

respectively. If $\theta^{\prime \prime}$ is another nearby generic structure and we fix the same representatives the gluing formula, extended to this situation (when we glue together two topological cylinders) yields

$$
G_{\theta, \theta^{\prime \prime}}(\psi)=G_{\theta, \theta^{\prime}}(\psi) \circ G_{\theta^{\prime}, \theta^{\prime \prime}}(\psi) .
$$

Thus to define our holomorphic bundle we decree that for each generic structure $\theta$ and choice of representatives $\psi$ the bundle has a canonical trivialisation over some neighbourhood $U_{\theta}$ of $l_{\theta}$ in the moduli space. Changing the representatives $\psi$ changes the trivialisation by multiplying by the diagonal metric $\Lambda_{\theta}$. On the the overlaps $U_{\theta} \cap U_{\theta^{\prime}}$ we use the maps $G_{\theta, \theta^{\prime}}(\psi)$ as transition functions. The gluing formula (37) is the cocycle condition 
giving the consistency of the set of transition functions, and the isomorphism $(-1)^{*}(\mathcal{E})=\mathcal{E}^{*}$ is induced by the fact that the solutions $\{\mathcal{S}\}$ of the equations defined by the 3 -forms $\rho,-\rho$ have an obvious identification.

Now to define the section $g_{Y_{1}}$ corresponding to $Y_{1}$ we work initially in a canonical trivialisation around $\theta$ and with a choice of the representatives $\psi$. By our hypothesis these can be extended to closed forms over $Y_{1}$, compatible with the product structure on the end. Then for an adapted bundle $E_{1}$ over $Y_{1}$ and a class $\psi$ in $H_{3}(Z)$ we define $\psi\left(E_{1}\right)$ in the same manner as before. We also have a limit $\mathcal{S}\left(E_{1}\right)$ and a number $n\left(E_{1}\right)$ Then we set

$$
\tilde{g}_{Y_{1}}(\psi)=\sum_{E_{1}} n\left(E_{1}\right) \exp \left(-\underline{\psi}\left(E_{1}\right)\right)<\mathcal{S}\left(E_{1}\right)>
$$

a $V$-valued holomorphic function on a neighbourhood of $l_{\theta}$. The gluing formula shows first that this yields a well-defined section $g_{Y_{1}}$ of $\mathcal{E}$-independent of the choice of $\theta$ and of $\underline{\psi}$ - and second that $g_{Y}=<g_{Y_{1}}, g_{Y_{2}}>$.

\section{Remarks}

1. Our bundle should have the property that its rank is equal to the DT invariant. We can think of the rank as the 0-degree component of the Chern character, and it might be that there is an extension of these ideas to a formula for all of ch $\mathcal{E}$. Note that Thomas' theory discusses a fixed Calabi-Yau manifold and there should be extensions of this which yield cohomology classes in the moduli space $\mathcal{M}$, using the universal family.

2. In this Section we have fixed attention on the $(6+1)$-dimensional theory but it is natural to wonder if there is some yet higher structure, associated to the 8-dimensional geometry. Roughly speaking, we would expect this to assign Floer groups to a compact $G_{2^{-}}$ manifold $Y$ and one would like some machinery to compute these when $Y=Y_{1} \cup_{Z} Y_{2}$. We make one observation in this direction. Suppose we have a vector bundle over a space $B$ given by transition functions $g_{\alpha, \beta}$ with respect to a cover $U_{\alpha}$ of $B$. Thus we have matrix entries $g_{\alpha, \beta, i, j}(z)$ which are functions of $z \in U_{\alpha} \cap U_{\beta}$ and

$$
g_{\alpha, \gamma, i, k}(z)=\sum_{j} g_{\alpha, \beta, i, j} g_{\beta, \gamma, j, k}
$$

on $U_{\alpha} \cap U_{\beta} \cap U_{\gamma}$. Now suppose we have a chain complex $C^{*}$ and a chain automorphism $T: C^{*} \rightarrow C^{*}$ so we have a Lefschetz number $L\left(C^{*}, T\right)=\sum(-1)^{i} \operatorname{Tr} T_{i}$. If we have a family of such pairs, parametrised by $z$, the Lefschetz number becomes a function of $z$. So we may envisage a structure given by chain complexes $C_{\alpha, \beta, i, j}^{*}$, with automorphisms, parametrised by $z \in U_{\alpha} \cap U_{\beta}$, such that on 
the triple overlaps

$$
C_{\alpha, \gamma, i, j, k} \sim \bigoplus_{j} \operatorname{Hom}\left(C_{\alpha, \beta, i, j}^{*}, C_{\beta, \gamma, j, k}^{*}\right),
$$

where $\sim$ is a suitable equivalence relation, at least as strong as chain homotopy equivalence, compatible with the automorphisms. Then the Lefschetz numbers of the chain complexes give the transition functions of a holomorphic bundle. Possibly there is some structure of this kind on the moduli space $\mathcal{M}$ which would refine the bundle $\mathcal{E}$, in the same way as the Floer homology of $G_{2}$-manifolds should refine the Casson invariant.

\section{Finite-dimensional analogue}

5.1. Morse-Novikov Theory. We begin with some basics. Take a manifold $A$, and a class $[\alpha] \in H^{1}(A, \mathbf{C})$. This gives a local system, which we may think of either as a rank 1 complex vector bundle on $A$ with flat connection $\alpha$, or as a representation (the monodromy of the connection)

$$
\begin{gathered}
\rho: \pi_{1}(A) \rightarrow \mathbf{C}^{*} \\
\rho(\gamma)=\exp \left(\int_{\gamma} \alpha\right)
\end{gathered}
$$

How do the cohomology groups $H^{*}(A, \rho)$ of this local system behave as we vary $[\alpha]$ ? The answer is that they form the fibres of a holomorphic sheaf $\mathcal{E}$ over the vector space $H:=H^{1}(A, \mathbf{C})$. We show this as follows. An element $\gamma \in \pi_{1}(A)$ gives a linear function on $H$. So we can let $\pi_{1}(A)$ act on the ring of holomorphic functions on $H$ by

$$
\gamma: f \mapsto e^{\gamma} f
$$

This is an action by module automorphisms, so we have a local system on $A$ whose fibre is the rank 1 free $\mathcal{O}_{H}$-module. The cohomology of this local system $\mathcal{E}:=H^{*}\left(A, \mathcal{O}_{H}\right)$ is then also an $\mathcal{O}_{H}$-module, i.e. a sheaf on $H$, and its fibre at the point $[\alpha]$ is the cohomology of the local system associated to $[\alpha]$.

We will now review Morse-Novikov theory in finite-dimensions, more specifically the reformulation due to Burghelea and Haller [2]. Let $\Theta$ be a closed real 1-form on a finite-dimensional Riemannian manifold $A$, and assume that $\Theta$ is "Morse", i.e. locally the differential of a Morse function. Then the pull-back of $\Theta$ to the universal cover $\tilde{A}$ is globally the differential of a Morse function, and we may form the cell-complex $C$ • given by the unstable manifolds of the zeroes of $\Theta$ on $\tilde{A}$ in the usual way. This cellcomplex is obviously periodic with respect the action of $\pi_{1}(A)$.

Now suppose we have a rank-1 complex local system on $A$ given by the class of some closed complex 1-form $\alpha$. Then we can use our periodic cellcomplex $C_{\bullet}$ in $\tilde{A}$ to produce a chain-complex that in good cases will calculate 
the cohomology of this local system. What we do is twist the differential on $C$. by $\alpha$ and then take $\pi_{1}(A)$ invariants. Assuming that $\Theta$ is in fact MorseSmale for the metric on $\tilde{A}$, we can describe this chain-complex explicitly in the following way: it has a basis given by the set $Z_{A}(\theta)=\{p\}$ of zeroes of $\Theta$ in $A$, and differential

$$
\partial_{\alpha} p=\sum_{i(q)=i(p)-1} \sum_{\gamma: p \rightarrow q} e^{\int_{\gamma} \alpha} q
$$

where $i(p)$ denotes the index of $p$, and $\gamma$ runs over all flow-lines of $\theta$ connecting $p$ and $q$. The differential clearly varies holomorphically in $\alpha$, so the homology of the chain complex is a holomorphic sheaf $\mathcal{E}$ on the space $\Omega_{c}^{1}$ of closed complex 1 -forms. If we choose a section $H \rightarrow \Omega_{c}^{1}$ (e.g. by using Hodge theory) we can pull-back and get a holomorphic sheaf on $H$. The fibre of this sheaf at $[\alpha]$ is the cohomology of the local system on $A$ given by $[\alpha]$.

There are two problems with this description. Firstly, since $\tilde{A}$ is noncompact the cell-complex $C$. may not cover the whole of $\tilde{A}$ (indeed $\theta$ may have no zeroes at all), so we can't guarantee that we are genuinely calculating the cohomology of the local system $[\alpha]$. Secondly, the sum (37) may not converge. It is shown in $[\mathbf{2}]$, that neither of these issues is a problem when $\alpha=s \theta$ for $s$ a complex number with $\operatorname{Re}(s)>\rho$, where $\rho \in[0, \infty]$ is some numerical invariant depending on $\theta$ and the metric. For this range of $\alpha$, the components of $\partial_{\alpha}$ do converge, and the chain-complex does calculate the cohomology of $[\alpha]$ correctly. The invariant $\rho$ is conjectured to be always finite, and in many examples is zero.

Let us suppose something stronger: that we get convergence and the correct cohomology in an open neighbourhood of the set $\operatorname{Re}(\alpha)=\theta$. This includes the set $\{\alpha=(1+i \lambda) \theta, \lambda \in \mathbf{R}\}$, where we do indeed get convergence and the right cohomology at least when $\rho<1$. If this holds, for any generic $\alpha_{0} \in \Omega_{c}^{1}$ we have an analytic way of constructing our sheaf $\mathcal{E}$ in a neighbourhood of $\alpha_{0}$. We set $\Theta=\operatorname{Re}\left(\alpha_{0}\right)$, then the Morse-Novikov chain-complex (38) constructed from $\theta$ will be valid in a neighbourhood of $\alpha_{0}$, so we may define $\mathcal{E}$ to be its homology.

Now consider the special situation in which $A$ is a complex manifold and $\Theta$ is the real part of a holomorphic 1-form. Then the indices of all critical points are equal and, in a generic situation there are no flow lines between critical points. For any fixed $\alpha$ the cohomology $H^{*}(A,[\alpha])$ is simply a vector space with one basis element for each zero of $\Theta$. The sheaf on $\mathcal{E}$ on $H^{1}(A, \mathbf{C})$ is locally free and we write $\mathcal{E}$ also for the corresponding holomorphic vector bundle.

We wish to apply this construction, formally, to the infinite-dimensional manifolds $\mathcal{X}$ arising from a Calabi-Yau threefold $Z$. Thus $\mathcal{X}$ is either the space of unitary connections with $F \wedge \omega^{2}=0$, modulo gauge equivalence, or the space of symplectic surfaces $\Sigma \subset Z$. Assume for simplicity that we are working with genuine Calabi-Yau structures. Then given a point $(I, \theta) \in \tilde{\mathcal{M}}$ 
we get an induced complex structure on $\mathcal{X}$ making it a Kahler manifold, and the Chern-Simons construction gives a holomorphic 1-form on $\mathcal{X}$ whose zeros correspond to stable holomorphic bundles or complex curves respectively. More generally, we have a map from $H_{3}(Z)$ to $H^{1}(\mathcal{X})$ and the ChernSimons construction gives a definite representative of this - a 1-form on $\mathcal{X}$ corresponding to a closed 3-form on $Z$.

Allowing the complex structure to vary, we get a map from $\tilde{\mathcal{M}}$ to $H^{1}(\mathcal{X} ; \mathbf{C})$, which is in fact a holomorphic embedding. So we should get a sheaf over $\tilde{\mathcal{M}}$ as the restriction of the twisted cohomology sheaf $\mathcal{E}$, assuming of course that we had made sense of the latter. Then everything is invariant under the mapping class group $\Gamma$ so we can descend to the moduli space $\mathcal{M}$.

It should now be fairly clear how our (conjectural) construction fits into this picture. Although we do not have any reason to believe that there is a cohomology sheaf over the whole of $H^{3}(Z, \mathbf{C})$ we can make sense of this over a neighbourhood of $\tilde{\mathcal{M}}$. An individual fibre, over a generic point, is rather uninteresting - having an almost-canonical basis. The interesting structure appears in the way these are fitted together into a vector bundle. To understand this we go back to our finite-dimensional situation and consider Morse theory on the universal cover.

Let $\theta$ again be a closed real 1-form on a finite-dimensional Riemannian manifold $A$, and (by abuse of notation) let $\theta$ also denote its pull-back to the universal cover $\tilde{A}$. Assume that $\theta$ is Morse-Smale, then we want to consider the resulting Morse complex for $\tilde{A}$.

Choose lifts $\tilde{p}$ of each zero $p \in A$ of $\theta$, so the zeros of $\theta$ on $\tilde{A}$ are

$$
Z_{\tilde{A}}(\theta)=\bigsqcup_{p}\left\{\gamma(\tilde{p}), \gamma \in \pi_{1}(A)\right\}
$$

This is a basis for the Morse complex. The set of flow-lines is $\pi_{1}(A)$-invariant, so when $i(q)=i(p)-1$ we may define $N_{p q}^{\gamma^{-1} \phi}$ to be the (signed) number of flow-lines between $\gamma(\tilde{p})$ and $\phi(\tilde{q})$, this depends only the product $\gamma^{-1} \phi$ in $\pi_{1}(A)$. Then the differential in the Morse complex is

$$
d(\gamma(\tilde{p}))=\sum_{i(q)=i(p)-1} \sum_{\phi \in \pi_{1}(A)} N_{p q}^{\gamma^{-1} \phi} \phi(\tilde{q})
$$

Since Morse homology is actually isomorphic to singular homology, it doesn't change as we vary $\theta$ and the metric. Floer observed in [10] that we can give an a priori proof of this fact, without reference to singular homology, by counting flow-lines in families. Suppose we have a suitably generic path $\left(\theta_{t}, g_{t}\right)$ of $\left(\pi_{1}(A)\right.$-invariant) closed 1-forms and metrics, where $\left(\theta_{0}, g_{0}\right)$ and $\left(\theta_{1}, g_{1}\right)$ are Morse-Smale. Then using some standard function we can define a 1 -form on $\tilde{A} \times[0,1]$ which has zeros

$$
\left\{\theta_{0}=0\right\} \times\{0\} \bigsqcup\left\{\theta_{1}=0\right\} \times\{1\}
$$


Let $M_{p q}^{\gamma^{-1} \phi}$ be the (signed) number of flow-lines

$$
\gamma(\tilde{p}) \times\{0\} \longrightarrow \phi(\tilde{q}) \times\{1\}
$$

where $i(p)=i(q)$, and define a linear map $\Psi$ between the Morse complexes for $\left(\theta_{0}, g_{0}\right)$ and $\left(\theta_{1}, g_{1}\right)$ by

$$
\Psi(\gamma(\tilde{p}))=\sum_{i(q)=i(p)} \sum_{\phi \in \pi_{1}(A)} M_{p q}^{\gamma^{-1} \phi} \phi(\tilde{q})
$$

Then it can be shown that $\Psi$ is a map of chain complexes, and that it is functorial with respect to composition of paths. Furthermore, if we have a homotopy between two such paths, then by a similar construction there is an induced homotopy between the corresponding chain maps. It follows that $\Psi$ is a homotopy equivalence. (Here we are assuming, in the finite-dimensional case, that we do not run into problems due to the noncompactness of $\tilde{A}$.)

Now suppose that $\theta_{0}=\boldsymbol{R e}\left(\alpha_{0}\right)$ and $\theta_{1}=\boldsymbol{R e}\left(\alpha_{1}\right)$ for some complex 1forms $\alpha_{0}$ and $\alpha_{1}$. We can use the above discussion to understand how the Morse-Novikov complexes for $\left(\alpha_{0}, g_{0}\right)$ and $\left(\alpha_{1}, g_{1}\right)$ are related. To do this, we need the following construction.

Let $\left(\theta_{0}, g_{0}\right)$ and $\left(\theta_{1}, g_{1}\right)$ be any two Morse-Smale pairs. Suppose, we have a $\pi_{1}(A)$-invariant linear map

$$
T:\left\langle Z_{\tilde{A}}\left(\theta_{0}\right)\right\rangle_{\mathbf{R}} \rightarrow\left\langle Z_{\tilde{A}}\left(\theta_{1}\right)\right\rangle_{\mathbf{R}}
$$

of the vector spaces underlying the corresponding Morse complexes. Then

$$
T: \gamma(\tilde{p}) \mapsto \sum_{q, \phi} T_{p q}^{\phi^{-1} \gamma} \phi(\tilde{q})
$$

for some set of real numbers $\left\{T_{p q}^{\gamma}\right\}$. Examples are the Morse differential $d$ when $\left(\theta_{0}, g_{0}\right)=\left(\theta_{1}, g_{1}\right)$, and the map $\Psi(39)$ when $\left(\theta_{0}, g_{0}\right) \neq\left(\theta_{1}, g_{1}\right)$. Then for any closed complex 1 -form $\alpha$ on $A$ we may formally define a map

$$
\hat{T}:\left\langle Z_{A}\left(\theta_{0}\right)\right\rangle_{\mathbf{C}} \rightarrow\left\langle Z_{A}\left(\theta_{1}\right)\right\rangle_{\mathbf{C}}
$$

by setting

$$
\hat{T}_{\alpha}(p)=\sum_{q, \gamma} T_{p q}^{\gamma} e^{\int_{\tilde{p}}^{\gamma(\tilde{q})} \alpha} q
$$

though of course this may not converge. It is easy to check that this process is (formally) a homomorphism, i.e. if we have three Morse-Smale pairs $\left(\theta_{0}, g_{0}\right),\left(\theta_{1}, g_{1}\right),\left(\theta_{2}, g_{2}\right)$ and maps

$$
\left\langle Z_{\tilde{A}}\left(\theta_{0}\right)\right\rangle_{\mathbf{R}} \stackrel{T}{\rightarrow}\left\langle Z_{\tilde{A}}\left(\theta_{1}\right)\right\rangle_{\mathbf{R}} \stackrel{U}{\rightarrow}\left\langle Z_{\tilde{A}}\left(\theta_{2}\right)\right\rangle_{\mathbf{R}}
$$


then

$$
\hat{T}_{\alpha} \hat{U}_{\alpha}=\widehat{T U}_{\alpha}
$$

Also applying this to the Morse differential we get

$$
\hat{d}_{\alpha}(p)=\sum_{i(q)=i(p)-1} \sum_{\gamma} N_{p q}^{\gamma} e^{\int_{\tilde{p}}^{\gamma(\tilde{q})} \alpha} q
$$

which is just the Morse-Novikov differential $\partial_{\alpha}$.

Now take two generic pairs $\left(\alpha_{0}, g_{0}\right),\left(\alpha_{1}, g_{1}\right)$ of closed complex 1-forms and metrics on $A$, and choose a generic path between them in the space of closed complex 1-forms and metrics. Take the real parts of all the 1-forms to get the same data in the space of closed real 1-forms and metrics. We get two Morse complexes with differentials $d^{0}$ and $d^{1}$, and a chain map $\Psi$ between them. Assume there is some neighbourhood $\mathcal{U} \subset \Omega_{c}^{1}$, containing $\alpha_{0}$ and $\alpha_{1}$, such that the maps $\hat{d}_{\alpha}^{0}, \hat{d}_{\alpha}^{1}$ and $\hat{\Psi}_{\alpha}$ converge for all $\alpha \in \mathcal{U}$. Then over $\mathcal{U}$ we have two Morse-Novikov complexes of holomorphic vector bundles on $\mathcal{U}$, given by the differentials $\hat{d}^{0}$ and $\hat{d}^{1}$. However, by (40) we know that $\hat{\Psi}$ is a holomorphic chain map between the two complexes.

If we have two such paths and a homotopy between them, we get two chain maps $\Psi^{1}$ and $\Psi^{2}$ between the Morse complexes, and chain-homotopy $\Xi$. Then, assuming everything converges in $\mathcal{U}$, we get two holomorphic chain maps $\hat{\Psi}^{1}$ and $\hat{\Psi}^{2}$ between our Morse-Novikov complexes on $\mathcal{U}$ and a holomorphic homotopy $\hat{\Xi}$ between them. Similarly, assuming that all the necessary maps converge in $\mathcal{U}$, we can show that our two Morse-Novikov complexes are homotopy equivalent.

In the model situation we are considering, the complex 1-forms on $A$ that are actually holomorphic, so at generic points the Morse-Novikov chain complexes have no differential. This means the homotopy equivalences $\hat{\Psi}$ are just isomorphisms, and that if $\hat{\Psi}^{1}$ and $\hat{\Psi}^{2}$ are homotopic maps then they are actually identical.

We now have a picture of how our bundle $\mathcal{E}$ on $H^{1}(A, \mathbf{C})$ is built up. Suppose we have a simply-connected region $\mathcal{U} \subset \mathcal{M}$ consisting of only two "chambers" $\mathcal{U}_{1}$ and $\mathcal{U}_{2}$ in which the Morse-Smale condition holds, separated by a wall on which it fails. Firstly, take two points $x, y \in \mathcal{U}_{1}$, and a path between them. The corresponding map $\Psi$ counts flow-lines in the family of 1 -forms and metrics given by the path, but since the Morse-Smale condition holds everywhere along the path we will just see a single flow-line from any zero of $\alpha_{x}$ to the corresponding zero of $\alpha_{y}$, so $\Psi$ is just the identity map. This means that $\mathcal{E}$ should be trivial over each region $\mathcal{U}_{1}$ and $\mathcal{U}_{2}$. However, if we have a path between $x \in \mathcal{U}_{1}$ and $y \in \mathcal{U}_{2}$ then the family will contain additional flow-lines arising from the "unexpected" flow-lines that occur on the wall, so the map $\Psi$ will be non-trivial. Passing to the holomorphic version, we get the transition function $\hat{\Psi}$ that we should use to patch $\mathcal{E}$ when we cross the wall. 
We can formulate this construction a little more precisely. Pick any $x \in$ $\mathcal{U}_{1}$ and $y \in \mathcal{U}_{2}$ and any path between them. The corresponding map $\Psi_{x y}$ is independent of the choice of path since $\mathcal{U}$ is simply-connected. Assume that $\hat{\Psi}_{x y}$ converges in all of $\mathcal{U}$. Let $V_{x}, V_{y}$ be the trivial bundles on $\mathcal{U}$ with fibres $\left\langle Z_{\mathcal{A}}\left(\alpha_{x}\right)\right\rangle_{\mathbf{C}}$ and $\left\langle Z_{\mathcal{A}}\left(\alpha_{y}\right)\right\rangle_{\mathbf{C}}$ respectively. Take another copy of each of these bundles, and denote them $V_{x y}:=V_{x}$ and $V_{y x}:=V_{y}$. Define

$$
\mathcal{E}_{\mathcal{U}}=\operatorname{coker}\left(V_{x y} \oplus V_{y x} \stackrel{\Phi}{\longrightarrow} V_{x} \oplus V_{y}\right)
$$

where

$$
\Phi=\left(\begin{array}{cc}
\operatorname{id} & \hat{\Psi}_{x y} \\
\hat{\Psi}_{x y}^{-1} & \text { id }
\end{array}\right)
$$

Then over $\mathcal{U}_{1}$ we have $\mathcal{E}_{\mathcal{U}} \cong V_{x}$ by projecting onto the first factor, and over $\mathcal{U}_{2}$ we have $\mathcal{E}_{\mathcal{U}} \cong V_{y}$ by projecting onto the second factor, but across the wall these isomorphisms differ by the transition function $\hat{\Psi}_{x y}$. This is precisely the vector bundle that we wanted.

In fact this picture of a finite wall-and-chamber decomposition is misleading. Since there are infinitely many homotopy classes in which nonMorse-Smale flow-lines can appear, we actually expect the set of walls to be dense in the parameter space $H^{1}(A, \mathbf{C})$. However, this construction adapts easily. Let $\mathcal{U}$ again be simply connected, but have possibly infinitely many walls in it. For every point $z \in \mathcal{U}$ that doesn't lie on a wall we let $V_{z}$ be the trivial bundle on $\mathcal{U}$ spanned by $Z_{\mathcal{A}}\left(\alpha_{z}\right)$, and for every distinct ordered pair $x, y$ of such points we take a copy $V_{x y}:=V_{x}$. For every pair $x, y$ we get a map $\Psi_{x y}$ of Morse complexes, and again this is independent of the choice of path between $x$ and $y$. Assume that $\hat{\Psi}_{x y}$ converges in all of $\mathcal{U}$ for all $x, y \in \mathcal{U}$. Define

$$
\mathcal{E}_{\mathcal{U}}=\operatorname{coker}\left(\bigoplus_{x, y} V_{x y} \stackrel{\Phi}{\longrightarrow} \bigoplus_{z} V_{z}\right)
$$

where the map $\Phi$ has components

$$
\delta_{x z} \mathrm{id}+\delta_{y z} \hat{\Psi}_{x y}: V_{x y} \rightarrow V_{z}
$$

At any generic point $z$ we have $\mathcal{E}_{\mathcal{U}} \cong V_{z}$ by projecting onto that factor, so in particular $\mathcal{E}_{\mathcal{U}}$ is a finite rank vector bundle.

5.2. Discussion. In our infinite-dimensional situation we interpret Floer's time dependent vector fields, used in constructing the chain homotopy, as almost- $G_{2}$ structures on topological cylinders. The count of flow lines for the time dependent vector field gives the count of $G_{2}$-instantons, and the Novikov series handle the passage to the universal cover. Then it should be clear how our definition of the holomorphic bundle over $\mathcal{M}$ matches up with the finite-dimensional discussion. To sum up, we interpret the holomorphic bundle $\mathcal{E}$ over $\mathcal{M}$ as the "middle-dimensional" cohomology of the infinite 
dimensional space $\mathcal{X}$, with coefficients in the local system over $\mathcal{X}$ defined by classes in $H^{3}(Z, \mathbf{C})$.

When Floer first introduced his theory he gave a different treatment of the parameter-dependence of his homology which was more explicit but technically more complicated. Later, he introduced the "time-dependent vector field" trick which gave a simpler approach, but the original method had the advantage of giving a more precise description of the chain homotopies. In a similar way we can give a more explicit description of the co-efficients which define the transition function of our holomorphic bundle. This is closer to the discussion in [6] and in [27]. The "Novikov" aspect of the theory is confusing here, so let us begin by considering a finite dimensional complex manifold $\tilde{A}$ and a proper holomorphic map $f: \tilde{A} \rightarrow \mathbf{C}$ with a finite number of critical values $z_{1}, \ldots, z_{N} \in \mathbf{C}$, where we suppose each $z_{i}$ corresponds to a unique critical point $A_{i} \in \tilde{A}$. The gradient curves of the function $\operatorname{Re}(f)$ on $\tilde{A}$ map under $f$ to line segments with fixed imaginary part. So if, as will generically be the case, the imaginary parts of the $z_{i}$ are all different there can be no gradient curves joining the critical points. However we are free to rotate the picture, multiplying $f$ by any complex number of modulus 1 . Given a pair $z_{i}, z_{j}$ we can choose this phase so that we are allowed to have gradient curves joining $A_{i}, A_{j}$, mapping to the line segment $\overline{z_{i} z_{j}}$ in $\mathbf{C}$, which generically will not contain any other $z_{k}$. The count of such gradient curves gives a number $N_{i j}$. This is the intersection number of the vanishing cycles of $f$ at the two points, when the fibre $f^{-1}(t)$ is transported along the segment $\overline{z_{i} z_{j}}$ so that the two vanishing cycles can be viewed as homology classes in the same space. Now suppose we have a generic 1-parameter family of such situations. Then in the family a third point $z_{k}$ may move across the segment $\overline{z_{i} z_{j}}$. When this happens the number $N_{i j}$ changes by $\pm N_{i k} N_{k j}$. Another way of expressing this is that we change the homotopy class of the path in $\mathbf{C} \backslash\left\{z_{1}, \ldots, z_{N}\right\}$ used to identify the fibres near $z_{i}$ and $z_{j}$. The parallel transport along the two paths differs by monodromy around $z_{k}$ : a Dehn twist in the vanishing cycle associated to $z_{k}$. Now given a generic $f$ we let $V_{f}$ be the vector space with basis symbols $\left\langle A_{i}\right\rangle$ associated to the critical points. As we move in a generic 1-parameter family from $f_{0}$ to $f_{1}$ say the $A_{i}$ move continuously so we have a naive, and rather trivial, isomorphism between $V_{f_{0}}$ and $V_{f_{1}}$ (depending on the path). We modify this in the following way. If in the family the critical point $z_{j}$ moves across the ray $\left\{z: \operatorname{Im}(z)=\mathbf{I m}\left(z_{i}\right), \boldsymbol{\operatorname { R e }}(z)>\boldsymbol{\operatorname { R e }}\left(z_{i}\right)\right\}$ then we map the basis element $\left\langle A_{i}\right\rangle$, before the crossing, to $\left\langle A_{i}\right\rangle \pm N_{i j}\left\langle A_{j}\right\rangle$, after the crossing.

Now replace the hypothesis that $f$ is proper and has a finite number of critical points by the situation we had before, where $\tilde{A}$ is the universal cover of some $A$, so there are a finite number of critical points up to the action of $H_{1}(A)$. The same procedure gives a more explicit recipe for the local trivialisation of the twisted cohomology bundle, except now we need to keep track of the relative homotopy class of paths between critical points, which brings in the Novikov series. Translated into our infinite dimensional picture 
this gives a way to describe the transition functions of our holomorphic bundle, using $G_{2}$-instantons on genuine tubes $\mathbf{R} \times Z$, exploiting the freedom to multiply the 3 -form $\theta$ by a phase.

With all of this discussion in place, we return to discuss our main hypothesis, that for generic points in the moduli space $\mathcal{M}$ all solutions are regular. This seems to be a serious restriction, not often satisfied in practice. However there is an obvious strategy for removing this restriction. We work with suitable generic perturbations of the Calabi-Yau structure, involving triples $\omega, \rho, \rho^{\prime}$ with $\omega \wedge \rho=0$. It is very reasonable to expect that for generic perturbations of this kind all solutions are regular. But as we explained in Section 3 we have then to give up the assumption that $\sigma$ is closed, so we get nonzero Fredholm indices for adapted bundles. But this just means that, in the finite-dimensional analogue, we need to compute twisted cohomology using a 1-form with zeros of different indices so we can have a nontrivial chain complex. What one would expect is that it is possible to define a collection of sheaves over the moduli space $\mathcal{M}_{R}$ which can be interpreted as the different twisted cohomology groups of $\mathcal{A} / / \mathcal{G}$. But we will not go into this further here.

Finally, we point out that, as explained by Burghelea and Haller, in the finite dimensional situation the twisted cohomology groups can be computed by a variant of Witten's complex, and described using differential forms satisfying Witten's deformation of the Hodge-Laplace equation. It could well be that the structure we are discussing in this article arise this way in Quantum Field Theory, and are perhaps well-known there.

\section{Interaction between gauge theory and calibrated geometry}

6.1. $G_{2}$-instantons and associative submanifolds. So far we have ignored the fundamental problem of compactness of moduli spaces. In this section we attempt to explore this and to get a glimpse of what modifications are required to take account of the problem.

Suppose that $\sigma_{i}$ is a sequence of $G_{2}$-structures on a fixed 7-manifold $Y$ with limit $\sigma$. Suppose we have corresponding sequence of $G_{2}$-instantons on a fixed $S U(l)$ bundle. Then, according to Tian, after taking a subsequence the connections converge off a set $P$ of Haussdorf dimension at most 3 and each three-dimensional component satisfies the associative condition, in a generalised sense. The sequence of 4 -forms $\operatorname{Tr}\left(F_{A_{i}}^{2}\right)$ allows us to attach a multiplicity to each 3-dimensional component. Let us suppose that in fact $P$ is a connected smooth associative submanifold. Roughly speaking, we expect that if the multiplicity is $k$ then transverse to $P$ the connections are modelled on instantons on $\mathbf{R}^{4}$ with $c_{2}=k$, and the behaviour as $i \rightarrow \infty$ mimics the familiar bubbling of instantons over 4-manifolds. We expect also that the singularity at $P$ in the limiting connection is removable, so that limiting connection extends to a smooth $G_{2}$-instanton over $Y[\mathbf{3 2}]$. In this section we discuss the converse question. Let $\sigma$ be a $G_{2}$ structure on $Y$ and $A$ be a 
$G_{2}$-instanton on a bundle $E$ over $Y$. Let $k$ be a positive integer and $P$ be an associative submanifold in $Y$. When does the triple $(A, P, k)$ appear as the limit of smooth $G_{2}$-instantons with respect to a sequence of deformations $\sigma_{i}$ of $\sigma$ ?

In this subsection we will explain that there is a natural candidate criterion for "bubbling" question. In particular when the gauge group is $S U(2)$ and when $k=1$ we will argue that this occurs if for some spin structure on $P$ the coupled Dirac operator on $\left.E\right|_{P}$, defined by the restriction of the connection $A$, has a nontrivial kernel. Several authors have considered related problems, mostly emphasising the similar question involving Cayley submanifolds and Spin(7)-instantons in 8-dimensions. Lewis proved an existence theorem for Spin(7)-instantons using a gluing construction, choosing a Cayley submanifold with very special properties [24]. Brendle considered the general question of existence of Yang-Mills connections [5], and Spin(7)-instantons in particular [4], but restricting attention to the case when (the analogue of) the integer $k$ above is 1 . The construction we want to explain here is due to Haydys $[\mathbf{1 4}],[\mathbf{1 5}]$, and related to ideas of Pidstrigatch $[\mathbf{2 8}]$ and Taubes [35]. We refer to the paper of Haydys [15] for a more complete account, and a discussion of various other interesting related matters.

To begin, suppose that $V$ is a quaternionic manifold, with a multiplication map

$$
\mu: T V \times \mathbf{H} \rightarrow T V .
$$

Then there is an elliptic "Fueter equation" for maps $f: \mathbf{R}^{3} \rightarrow V$ which is

$$
I \frac{\partial f}{\partial y_{1}}+J \frac{\partial f}{\partial y_{2}}+K \frac{\partial f}{\partial y_{3}}=0 .
$$

In the case when $V$ is $\mathbf{H}$ this is just the Dirac equation for a spinor field. (There is a similar equation for maps from $\mathbf{R}^{4}$ to $V$, but we will emphasise the 3-dimensional version.) Now suppose that there is an action of $S U(2)$ on $V$ permuting the $I, J, K$. More precisely, this means that $\mu$ in (41) is an $S U(2)$-equivariant map, for the induced action of $S U(2)$ on $T V$ and the standard action by automorphisms of the quaternions. Let $P$ be an oriented Riemannian 3-manifold with a spin structure and $\mathrm{Fr} \rightarrow P$ the corresponding principal $S U(2)$ bundle. Then we can form the associated bundle

$$
\underline{V}=\operatorname{Fr} \times_{S U(2)} V .
$$

For each point $y \in P$ there is an obvious way to make $\mathbf{R} \oplus T P_{y}$ into an algebra $H_{y}$, isomorphic to $\mathbf{H}$ but not canonically so. Our hypotheses imply that there is a natural $H_{y}$ structure on the tangent bundle of the fibre $\underline{V}_{y}$. Thus there is a Fueter equation for sections of $\underline{V}$,

$$
\sum_{i} e_{i} \nabla_{i} s=0
$$


where $e_{i}$ is any orthonormal frame in $T P_{y}$ and $\nabla_{i}$ denotes the "vertical part" of the derivative of the section, defined using the horizontal subspace induced from the Levi-Civita connection. Slightly more generally still, suppose that $G$ is another Lie group and there is an action of $G \times S U(2)$ on $V$, where now $G$ preserves the quaternionic structure. Let $Q \rightarrow P$ be a $G$-bundle with connection, so we have a principal $G \times S U(2)$ bundle $Q \times{ }_{P} F r$ over $Y$. Then we can form an associated fibre bundle

$$
\left(Q \times{ }_{P} F r\right) \times{ }_{G \times S U(2)} V,
$$

and, as Haydys observed [14], there is an intrinsic Fueter equation for sections of this bundle over $P$.

With this background in place, we return to consider an associative submanifold $P \subset Y$. To explain the basic idea, we begin with the model case when $P, Y$ are flat, so we can take local co-ordinates $y_{1}, y_{2}, y_{3}, x_{1}, x_{2}, x_{3}, x_{4}$ in which

$$
\sigma=d x_{1} d x_{2} d x_{3} d x_{4}+\sum_{i, j, k \text { cyclic }} d y_{i} d y_{j} \omega_{k}
$$

where $\omega_{i}$ is a standard basis for $\Lambda^{+} \mathbf{R}^{4}$. For $\epsilon>0$ let $\sigma_{\epsilon}$ be $\epsilon^{-2}$ times the pull-back of $\sigma$ under the map which multiplies the $x_{a}$ co-ordinates by $\epsilon$. Thus

$$
\sigma_{\epsilon}=\epsilon^{2} d x_{1} d x_{2} d x_{3} d x_{4}+\sum d y_{i} d y_{j} \omega_{k} .
$$

We have a limit $\sigma^{*}=\sum d y_{i} d y_{j} \omega_{k}$ which is not a positive form, but we can still consider the equation $F \wedge \sigma^{*}=0$, which we refer to as the $\sigma^{*}$-instanton equation.

Let $N \rightarrow P$ be the normal bundle of $P \subset Y$. The $G_{2}$-structure induces a bundle isomorphism $\Lambda^{+} N \rightarrow T P$, which one finds is covariant constant with respect to the standard induced connections on $T P, N$. Fix a spin structure on $P$. Then we get another complex vector bundle $U \rightarrow P$, with a connection and structure group $S U(2)$, such that $N$ is canonically identified with the real part of $U \otimes_{\mathbf{C}} S$. Using the connection on $N$ we get a canonical 3 -form $\sigma^{*}$ on the total space of $N$. Let exp be the exponential map from a neighbourhood of the zero-section in $N$ to $Y$, let $\exp _{\epsilon}(\xi)=\exp (\epsilon \xi)$ and let $\sigma_{\epsilon}$ be the 4-form $\epsilon^{-2} \exp _{\epsilon}^{*}(\sigma)$. Then one can see that the limit of $\sigma_{\epsilon}$ as $\epsilon$ tends to zero is $\sigma^{*}$. We can define $\sigma^{*}$-instantons: connections on bundles over the total space $N$, as above and it clearly reasonable to expect that these are the blow-up limits of sequences of connections developing a singularity along $P$.

Now we want to bring in a $G_{2}$-instanton connection $A$ on another $S U(l)$ bundle $E$ over $Y$. This defines a connection $\left.A\right|_{P}$ on the restriction $\left.E\right|_{P}$. Let $N_{\infty}$ be the 4 -sphere bundle over $P$ obtained by adjoining a section at infinity to the $\mathbf{R}^{4}$-bundle $N$. W define a $\sigma^{*}$-instanton on $N$ with asymptotic limit $\left.A\right|_{P}$ to be given by a connection $\mathbf{A}$ on a bundle $\mathbf{E} \rightarrow N_{\infty}$ which satisfies the $\sigma^{*}$-instanton equations on the dense subset $N \subset N_{\infty}$ and such that the restriction of $\mathbf{A}$ to the infinity section is equivalent to $\left.A\right|_{P}$. Note that this data defines an integer Chern class $k$, given by the restriction of $\mathbf{E}$ to any 
4-sphere fibre. We expect that a triple $(A, P, k)$ can only occur as the limit of a sequence of $G_{2}$-instantons, for nearby structures, if there is a solution of the $\sigma^{*}$-instanton equation with Chern class $k$ and asymptotic limit $\left.A\right|_{P}$.

Let $M=M_{k, l}$ be the moduli space of "framed" $S U(l)$ instantons of Chern class $k$ over $S^{4}=\mathbf{R}^{4} \cup\{\infty\}$. By a framing we mean a trivialisation of the fibre over $\infty$. We write $\mathbf{R}^{4}$ as the real part of $S^{+} \otimes S^{-}$where $S^{+}, S^{-}$are the spin spaces. Then there is a natural action of the group $S U(2) \times S U(2) \times S U(l)$ on $M$, where the two $S U(2)$ factors act on $S^{+}, S^{-}$, and $S U(l)$ acts on the framing at infinity. There is a quaternionic structure on $M$ which is preserved by $S U(l)$ and the second copy of $S U(2)$ and permuted by the first copy of $S U(2)$. So we are in the situation above, with $G=S U(2) \times S U(l)$. Given our $S U(l)$-bundle $\left.\mathbf{E}\right|_{P}$ and our $S U(2)$-bundle $U \rightarrow P$ we form a $G$-bundle as the fibre product and then we get a bundle $\underline{M} \rightarrow Y$ with fibre $M$ and a Fueter equation for sections of $\underline{M}$.

TheOREM 1. (Haydys [15]) There is a one-to-one correspondence between solutions of the $\sigma^{*}$ instanton equation with Chern class $k$ and asymptotic limit $\left.A\right|_{P}$ and sections of the bundle $\underline{M}$ over $P$ which satisfy the Fueter equation.

This can be thought of a variant of the "adiabatic limit" for $\operatorname{Spin}(7)$ instantons over products discussed in [6]. In the case when $k=1$ the Fueter equation appears in [4] as a "balancing condition". It is natural to expect that the equation in [5] associated to general Yang-Mills solutions can be interpreted as the equation defining a harmonic section of the bundle $\underline{M}$, as in $[\mathbf{1 6}]$.

We use the connection on $N$ to split the tangent space of $N$ into horizontal and vertical subspaces, isomorphic to $T P$ and $(U \otimes S)_{\mathbf{R}}$ respectively. With respect to this splitting, $F(A) \wedge \sigma^{*}$ has two components, say $F_{1}, F_{2}$ where $F_{1}$ takes values in the normal bundle of $P$ and $F_{2}$ in the tangent bundle (both lifted to $N$ and tensored with the bundle of Lie algebras). The $\sigma^{*}$-instanton equation thus splits into two separate conditions. We show that there is a one-to-one correspondence between

- connections A over $N_{\infty}$ isomorphic to $A$ over the section at infinity and satisfying $F_{2}=0$

- smooth sections of $\underline{M} \rightarrow Y$.

Then we show that the further condition $F_{1}=0$ is equivalent to the Fueter equation.

The condition $F_{2}=0$ just asserts that the restriction of $\mathbf{A}$ to each fibre of $N$ is an anti-self-dual connection. There is a tautological bundle over $M \times S^{4}$ which is equivariant for an action of $S U(2) \times S U(2) \times S U(l)$ and which has a fixed trivialisation over $M \times\{\infty\}$. On this bundle we have a standard connection which restricts tautologically to the $S^{4}$ slices and which is compatible with the trivialisation over $M \times\{\infty\}$. Using the group action we construct a bundle $\tilde{E}$ over the pull-back $\pi^{*}(\underline{M})$ of $\underline{M}$ to $N_{\infty}$, which 
can also be viewed as a bundle over $P$ with fibre $S^{4} \times M$. The connections on $T P, U$ and $E$ induce a natural connection $\mathbf{A}_{0}$ on $\tilde{E}$. A section $s$ of $\underline{M}$ induces a section $\bar{s}$ of $\pi^{*}(\underline{M})$ and we have a connection $A_{s}=\bar{s}^{*}\left(\mathbf{A}_{0}\right)$ on a bundle $\bar{s}^{*}(\tilde{E})$ over $N_{\infty}$. The stated properties of the connection on the universal bundle imply that $A_{s}$ is isomorphic to $A$ over the section at infinity and satisfies the condition $F_{2}=0$, simply because the connection on the universal bundle is anti-self-dual on each $\mathbf{R}^{4}$ slice in $M \times \mathbf{R}^{4}$. Conversely, it is a straightforward formal exercise to check that all such connections arise in this way.

The remaining task is to match up the curvature condition $F_{1}=0$ with the Fueter equation. For this we should recall some more of the theory of instantons over $\mathbf{R}^{4}$. We can regard $\mathbf{R} \oplus \Lambda^{+}$as an algebra $H$. Of course, as before, $H$ is isomorphic to the quaternions but we prefer not to fix such an isomorphism. Then $H$ acts naturally by multiplication on itself and also acts on $\mathbf{R}^{4}$. Let $A$ be a finite energy instanton over $\mathbf{R}^{4}$. We have a defomation complex $\Omega^{0} \stackrel{d_{A}}{\rightarrow} \Omega^{1} \stackrel{d_{A}^{+}}{\rightarrow} \Omega^{+}$and an elliptic operator $d_{A}^{*} \oplus d_{A}^{+}: \Omega^{1} \rightarrow \Omega^{0} \oplus \Omega^{+}$. The tangent space of the moduli space $M$ at $A$ can be identified with the $L^{2}$ solutions $a$ of $\left(d_{A}^{*} \oplus d_{A}^{+}\right) a=0[\mathbf{3 3}]$. The crucial points are

- $d_{A}^{*} \oplus d_{A}^{+}$commutes with the $H$ action induced by the actions on $\mathbf{R} \oplus \Lambda^{+}$and $\mathbf{R}^{4}$. Thus we get an action of $H$ on the tangent space of $M$, which is just the quaternionic structure mentioned before.

- The component of the curvature of the connection $A$ on the universal bundle in $T^{*} M \otimes T^{*} \mathbf{R}^{4}=\operatorname{Hom}\left(T M, T^{*} \mathbf{R}^{4}\right)$ is the tautological map given by evaluating $a \in T M$ at a point in $\mathbf{R}^{4}$. In particular this commutes with the action of $H$.

Now we work at a fixed point in $P$ and fix an orthonormal basis $e_{i}$ for the tangent space of $P$ at this point. Identifying the fibre of $N$ at this point with $\mathbf{R}^{4}$ we get a basis $\omega_{i}$ of $\Lambda^{+}$. Suppose we have a section $s$ of $\underline{M} \rightarrow P$. With respect to the given connections this has a covariant derivative, with three components $a_{1}, a_{2}, a_{3} \in T M$ corresponding to the tangent vectors $e_{i}$. The Fueter equation is $\sum \omega_{i}\left(a_{i}\right)=0$. By the second observation above this implies that for each point $x \in \mathbf{R}^{4}$

$$
\sum \omega_{i}\left(F_{a_{i}},\right)=0
$$

where $\left(F_{a_{i}},\right)$ is the bundle-valued 1 -form on $\mathbf{R}^{4}$ obtained by pairing the curvature of the universal bundle with $a_{i} \in T M$. Unravelling the definitions one sees that the left hand side of this equation is precisely the component $F_{1}$ of the curvature. Thus a solution of the Fueter equation does yield a solution of the $\sigma^{*}$-instanton equation and the only remaining thing is to see that there are no other solutions.

Suppose we have any solution $\mathbf{A}$ of the equation $F_{2}=0$, isomorphic to $A$ over the infinity section. Restriction to the fibres of $N$ defines a section $s$ of $\underline{M}$ and $\mathbf{A}$ must agree with $A_{s}$ in the fibre direction. Thus the only 
possibility is that $\mathbf{A}=A_{s}+\Phi$ where $\Phi$ is a bundle-valued 1-form vanishing in the fibre direction. If we fix a point in $P$ and a basis $e_{i}$ as above then $\Phi$ has three components $\phi_{i}$ which are sections of the adjoint bundle over $\mathbf{R}^{4}$. If $a_{i}$ are the derivatives of $s$, as above, then the curvature condition $F_{1}=0$ becomes

$$
\omega_{i}\left(a_{i}+d_{A} \phi_{i}\right)=0 .
$$

The hypothesis on the connection over the infinity section is equivalent to the condition that $\left|\phi_{i}(x)\right| \rightarrow 0$ as $x \in \mathbf{R}^{4}$ tends to infinity. Thus what we need to show is that in this situation all the $\phi_{i}$ vanish. Now write $\Phi=\sum \phi_{i} \omega_{i}$, a bundle-valued self-dual 2-form over $\mathbf{R}^{4}$. The equation (44) is equivalent to

$$
d_{A}^{*} \Phi=\sum \omega_{i}\left(a_{i}\right) .
$$

Since $d_{A}^{+} \omega_{i}\left(a_{i}\right)=0$ we have the identity $d_{A}^{+} d_{A}^{*} \Phi=0$. The Weitzenbock formula in this situation tells us that

$$
d_{A}^{+} d_{A}^{*} \Phi=\sum_{i}\left(d_{A}^{*} d_{A} \phi_{i}\right) \omega_{i}
$$

(This uses the fact that $A$ is an anti-self-dual connection.) So we deduce that $d_{A}^{*} d_{A} \phi_{i}=0$ and then the maximum principle implies that $\phi$ vanishes, since $\left|\phi_{i}\right|$ tends to zero at infinity. This completes the proof of the theorem.

There is a standard map from $M$ to $\mathbf{R}^{4}$ which takes a connection to the centre of mass of its curvature density $|F|^{2}$ and the derivative of this map is $H$-linear. In fact $M$ is a product $M^{\prime} \times \mathbf{R}^{4}$, where $M^{\prime}$ is the "centred" moduli space. It follows that there is a bundle map from $\underline{M}$ to $N$ which takes solutions of the Fueter equation for sections of $\underline{M}$ to sections of the corresponding equation for sections of $N$. The latter is just the linear Dirac equation appearing in the theory of deformations of the associative submanifold $P$. We assume $P$ is "regular" so this equation has no non-zero solution. This means that we can replace the instanton moduli space $M$ by the centred moduli space $M^{\prime}$ throughout the discussion above. Let us consider the case when $l=2$ and $k=1$. Then, up to translation and dilation of $\mathbf{R}^{4}$ and gauge equivalence, there is just one instanton which is the standard connection on the negative spin bundle over $S^{4}$. The framed moduli space can naturally be written as

$$
M^{\prime}=\left(S^{+} \backslash\{0\}\right) / \pm 1,
$$

where $S^{+}$is the spin space, and this is compatible with the quaternionic structure. (The quotient by \pm 1 comes from the centre of $S U(2)$.) Tracing through the definitions we find that

$$
\underline{M}^{\prime}=\left(\left(S^{+} \otimes E\right)_{\mathbf{R}} \backslash \underline{0}\right) / \pm 1 .
$$

where $S^{+}$is the spin bundle over $P$ and $\underline{0}$ denotes the zero-section. Note that the bundle $\underline{M}^{\prime}$ does not depend on the choice of a spin structure on $Y$ but if we have a section of $\underline{M}^{\prime}$ there is a unique choice of spin structure 
for which this lifts to a section of $\left(S^{+} \otimes E\right)_{\mathbf{R}}$. Making this choice, we see that solutions of the Fueter equation correspond (up to \pm 1 ) to solutions of the Dirac equation for sections of $E$-valued spinors over $P$, using the Dirac operator coupled to $A$. Thus, in sum we expect that the condition that a pair $P, A$ appear as the limit of $G_{2}$-instantons, with multiplicity $k=1$, is that there is a nontrivial harmonic spinor for the restriction $\left.A\right|_{P}$, for some choice of spin structure on $P$. (Note that we are assuming here that the harmonic spinor does not vanish, but this should be true generically for dimensional reasons. A case when the harmonic spinor vanishes somewhere would require further analysis.)

We could carry out the same discussion for complex curves in a CalabiYau 3-fold and Hermitian-Yang-Mills connections (solutions of (22)), or embed this in the situation above by taking the product with a circle. But in this 6-dimensional case there is an alternative, algebro-geometric, point of view, which leads to the same conclusion. Let $\Sigma$ be a smooth curve in a Calabi-Yau 3-fold $Z_{0}$. Suppose $\pi: \mathcal{Z} \rightarrow \Delta$ is a deformation of $Z_{0}$, where $\Delta$ is a disc in $\mathbf{C}$. Thus $\Sigma$ can be viewed as a curve in the central fibre $\pi^{-1}(0) \subset \mathcal{Z}$. Blow up this curve to get $\tilde{\pi}: \tilde{\mathcal{Z}} \rightarrow \Delta$. Then

$$
\tilde{\pi}^{-1}(0)=V \cup_{D} \tilde{Z}_{0},
$$

where $\tilde{Z}_{0}$ is the blow-up of $Z_{0}$ along $\Sigma, D \subset \tilde{Z}_{0}$ is the exceptional divisor, which is a $\mathbf{C} \mathbf{P}^{1}$ bundle over $\Sigma$, and $V$ is a $\mathbf{C P}^{2}$ bundle over $\Sigma$ which contains a copy of $D$. If we have a holomorphic bundle $\mathbf{E}$ over $\tilde{\mathcal{Z}}$, restriction to fibres gives a family of bundles $E_{t}$ over the deformations $Z_{t}=\pi^{-1}(t)$ for non-zero $t$ but if the restriction of $\mathbf{E}$ to the $\mathbf{C P}^{2}$ fibres in $V$ is non-trivial this family will not extend to give a bundle over $Z_{0}$. One expects this to give the algebro-geometric description of a family of Hermitian-Yang-Mills connections developing a singularity along $\Sigma$. The analogue of the connection $A$ in the discussion above is furnished by the restriction of $\mathbf{E}$ to $\tilde{Z}_{0} \subset \tilde{\pi}^{-1}(0)$, which we assume to be the lift of a bundle $E$ over $Z_{0}$. The algebro-geometric analogue of the question we have discussed above is to ask: given $\Sigma, E, k$ when is there a bundle over $\tilde{\pi}^{-1}(0)$ which is isomorphic to the pull-back of $E$ over $\tilde{Z}_{0}$ and has $c_{2}=k$ on the $\mathbf{C P}^{2}$ fibres in $V$. It is a straightforward algebraic geometry exercise to show that when $k=1$ this occurs precisely when there is a non-vanishing holomorphic section of $E \otimes K_{\Sigma}^{1 / 2}$ over $\Sigma$, for some choice of spin structure $K_{\Sigma}^{1 / 2}$.

6.2. Implications. The Dirac operator on $E$-valued spinors over $P$ is naturally a real operator and we expect to encounter a zero eigenvalue in real codimension 1 . Thus it seems likely that a naive count of $G_{2}$-instantons will not yield an invariant. What one would expect is needed is a count which includes triples $(A, P, k)$ of a connection on a different bundle and an associative submanifold $P$, thought of as having multiplicity $k$. We should count these with some weight $W(A, P, k)$. For example with bundles of rank 
$l=2$ and when $k=1$ we would need some way to determine the weight $W$ so that in a generic 1-parameter family it changes by \pm 1 when one eigenvalue of the Dirac operator crosses 0 (and a prerequisite for understanding the signs would be to develop a theory of orientations and signs in the "naive" theory, which we have not discussed). This is much the same issue involved in regularising the "dimension" in the ordinary Floer theory, as we discussed in Section 4.1. Formally $W$ could be given by the $\pm 1 / 2$ where the sign is determined by the parity of the "number of negative eigenvalues" of the coupled Dirac operator on $P$. It seems possible that this can be done, using the theory of spectral flow.

For larger values of $k$ new issues arise, since the contribution from $P$ involves an essentially non-linear problem. We should still expect to encounter solutions in real codimension 1 . The reason is that there is a dilation action on $M$ and hence on $\underline{M}$ which preserves the quaternionic structure. Thus a single solution of the Fueter equation generates a 1-dimensional family, by dilation. The linearisation of the Fueter equation has Fredholm index 0 but this dilation action implies that, in a family, we expect to encounter solutions in real codimension 1 , just as for the linear Dirac operator. Given any section $s$ of $\underline{M}$, let $\hat{s}$ denote the vertical vector field defined by the infinitesimal dilation action, and let $\mathcal{D}(s)$ be the expression appearing in the Fueter equation, which is also a vertical vector field. Then we have a nonlinear eigenvalue equation

$$
\mathcal{D}(s)=\lambda \hat{s},
$$

for sections $s$, generalising the eigenvalue equation for the Dirac operator. (In [13], Section 3.4, Haydys develops a more general theory of these eigenvalue equations, in terms of a "Swann bundle".) Of course we have a solution of the Fueter equation just when there is a zero eigenvalue. So it seems that one needs an extension of the theory of spectral flow which would enable one to define the weight $W(A, P, k)$ by a regularisation of the "number of negative eigenvalues" for this nonlinear problem. If one seeks, more ambitiously, to construct a Floer theory in 7-dimensions then it seems likely that one would have to assign a Floer group (or, perhaps better, chain complex) to $(A, P, k)$, giving the contribution to the overall Floer homology (and with Euler characteristic $W(A, P, k)$ ). This may be related to recent work of Hohloch, Noetal and Salamon [17]. We could think of a "completed" space of connections with a point having a neighborhood modelled on the product of a Hilbert space with a cone over a space $\mathcal{L}$, where $\mathcal{L}$ is the space of sections of $\underline{M}$, modulo dilation. In a finite-dimensional analogue the contribution of this point in a Morse theory description of the homology of the total space will involve the theory of the Conley index; the homology of $\mathcal{L}$ and various subsets. It is possible that there is a "Floer-analogue" of this which can be formulated in terms of the solutions of the eigenvalue equation, and "flow lines" between them. 
Even leaving aside this complication in 7-dimensions, of bubbling along an associative submanifold, it seems likely that the naive count of solutions in 6 dimensions is not the right thing to consider for the purposes of developing a $6+1$ dimensional theory, including the conjectural holomorphic bundle $\mathcal{E}$ we have discussed. Consider a nonsingular $G_{2}$ instanton over a topological cylinder $\mathbf{R} \times Z$. Restriction to slices $\{t\} \times Z$ gives a family of connections over $Z$. One can imagine a case when as $t \rightarrow \infty$ these connections develop a singularity along $\Sigma \subset Z$. This phenomenon suggests that one would have to take account of pairs $(E, \Sigma)$ in $Z$ in order to have the correct gluing identities. This fits in with the fact that the Thomas' algebro-geometric approach to the holomorphic Casson invariant includes contributions from sheaves, not just bundles. There seems to be a lot of scope for work relating the algebro-geometric and differential-geometric points of view.

6.3. Codimension- 3 theories and monopoles. This subsection is rather more speculative. Given a noncompact Riemannian 3-manifold $B$ with an end of a suitable kind one can study the Bogomolny monopole equation $F(A)=* d_{A} \Phi$ for a connection $A$ and section $\Phi$ of the adjoint bundle. We will just consider the case of structure group $S U(2)$. Solutions correspond to translation-invariant instantons on $\mathbf{R} \times B$. One also imposes asymptotic conditions on the ends of $B$, the most important being that $|\Phi| \rightarrow r^{-1}$ at infinity, where $r>0$ is fixed. The most familiar case is when $B=\mathbf{R}^{3}$ and then one can reduce to the case when $r=1$ by scaling the metric. But in general $r$ will be a genuine parameter and it will not be possible to identify solutions for different values of $r$. The references $[\mathbf{3}],[\mathbf{9}],[\mathbf{1 8}],[\mathbf{1}]$, and many others, give more details about this monopole theory.

Now suppose we have a noncompact $G_{2}$-manifold $Y$. We can study the analogous equation (25) on $Y$ with the asymptotic condition $|u| \rightarrow r^{-1}$ at infinity. Let us imagine that, for manifolds $Y$ with an end of a suitable kind, we can find a set-up which leads to a Fredholm problem and to invariants, which would be numbers in the case of index zero. Then we could study the behaviour of solutions as the parameter $r$ varies, in particular as $r \rightarrow$ 0 . We can find plausible models for this based on compact co-associative submanifolds $Q \subset Y$, in much the same way as we modelled the blow-up behaviour around associative submanifolds. For simplicity consider first the flat case, so we have standard co-ordinates $x_{a}$ on $Q$ and $y_{i}$ normal to $Q$. The equations (25) can be written very schematically as

$$
\nabla_{\underline{y}} u=F_{\underline{y y}}+F_{\underline{x x}} \quad \nabla_{\underline{x}} u=F_{\underline{x} \underline{y}} .
$$

If we change variables, replacing $y_{i}$ by $r y_{i}$ and $u$ by $r^{-1} u$, then take the limit as $r$ tends to zero, we get a limiting equations

$$
\nabla_{\underline{y}} u=F_{\underline{y y}} \quad \nabla_{\underline{x}} u=F_{\underline{x} \underline{y}} .
$$


The first of these equations is the Bogomolony monopole equation on each $\mathbf{R}^{3}$ slice normal to $Q$ and the second equation is the Fueter equation for the resulting map from $Q$ to the moduli space of monopoles on $\mathbf{R}^{3}$. To say this more systematically and generally, recall that for each integer $k>0$ we have a moduli space $\mathrm{Mon}_{k}$ of "centred" monopoles of charge $k$ on $\mathbf{R}^{3}$. (The charge is a topological invariant given by the degree of $\phi$ over a large sphere, in any trivialisation of the bundle.) This moduli space is a hyperkahler manifold of real dimension $4(k-1)$ and the rotations of $\mathbf{R}^{3}$ act on $\mathrm{Mon}_{k}$, permuting the complex structures. Given any compact coassociative submanifold $Q \subset Y$ we can form a bundle $\underline{\operatorname{Mon}}_{k} \rightarrow Q$ with fibre $\operatorname{Mon}_{k}$ much as before and there is a Fueter equation for sections of $\underline{\mathrm{Mon}}_{k}$. We interpret solutions of this Fueter equation as possible asymptotic limits for sequences of solutions of (25) over $Y$ as the parameter $r$ tends to 0 . These solutions are localised around $Q$ and should be very close to reducible away from $Q$, with the structure group reduced to $S^{1} \subset S U(2)$ by the "Higgs field" $u$ (which would be almost covariant constant away from $Q$ ).

We could make exactly the same discussion for a noncompact Calabi-Yau manifold $Z^{6}$, and we will now switch our focus to this case as it is simpler. We suppose the elementary topology of the set-up allows us to associate a class in $H_{3}(Z)$ to our problem, analogous to the monopole charge in 3-dimensions. Then for each class $\kappa \in H_{3}(Z)$ we expect to have a numerical invariant $n_{\kappa}$, counting solutions of (22). Our model for the asymptotic behaviour involves a special Lagrangian submanifold $P \subset Z$. For each $k$ we form a monopole bundle over $P$ and we have a Fueter equation for sections. This has index 0 and we imagine we can define a number $w(k, P)$ by counting the solutions. Then we could hope to express the number $n_{\kappa}$ as some kind of count of special Lagrangian submanifolds $P$, weighted by the numbers $w(k, P)$. The simplest guess is a formula of the shape

$$
n_{\kappa}=\sum_{\kappa=\sum k_{i}\left[P_{i}\right]} \prod_{i} w\left(k_{i}, P_{i}\right)
$$

This may be a rather crude approximation to the truth of the matter, because we have not discussed what happens when two special Lagrangians intersect. But in any case we could hope that there is some way of computing $n_{\kappa}$ from data localised around special Lagrangian submanifolds.

This picture, if it stands up to closer scrutiny, is rather similar to Taubes' relation between the Seiberg-Witten and Gromov invariants of a symplectic four-manifold $W[\mathbf{3 4}]$. The moduli space of vortices on $\mathbf{R}^{2}$ would play the role in that case that the moduli space of monopoles does above. For a given "charge" $k$ the moduli space of vortices is just the k-fold symmetric product of $\mathbf{R}^{2}[\mathbf{1 8}]$. When the ambient space is actually a complex surface we arrive in the realm of ordinary algebraic geometry. Given a curve $\Sigma \subset W$ a section of the appropriate "vortex bundle" corresponds to an infinitesimal deformation of the order $k$ formal neighbourhood of $\Sigma$, as a subscheme of $W$. 
All of this discussion assumes that we can indeed find the correct set-up to define numerical invariants $n_{\kappa}$. The point we want to emphasise is that, if this can be done, one might hope that these are easier to define than the counts of special Lagrangians. Thus, by analogy, the Seiberg-Witten invariants of a 4-manifold are much easier to define than the Gromov invariants. Then we could take (47) as the definition, or a guide to the definition, of an invariant counting special Lagrangian submanifolds.

\section{References}

[1] Atiyah, M.F. and Hitchin, N.J. The geometry and dynamics of magnetic monopoles Princeton U.P. (1988).

[2] Burghelea, D. and Haller, S. On the topology and analysis of a closed 1-form, I (Novikov's theory revisited) In:Essays on geometry and relatd topics, Vol. 1 133-175 Monogr. Enseign. Math. 38 Geneva (2001).

[3] Braam, P.J. Magnetic monopoles on 3-manifolds Jour. Differential Geometry 30 425464 (1989).

[4] Brendle, S. Complex anti-self-dual instantons and Cayley submanifolds arxiv DG/0302094.

[5] Brendle, S. On the construction of solutions to the Yang-Mills equations in higher dimensions arxiv DG/0302093.

[6] Donaldson, S.K. and Thomas, R.P. Gauge Theory in higher dimensions In: The Geometric Universe, Huggett et al. Eds. Oxford U.P. (1998).

[7] Donaldson, S.K. Moment maps and diffeomorphisms Asian J. Math. 3 1-15 (1999).

[8] Donaldson, S.K. Floer homology groups in Yang-Mills theory Cambridge U.P. (2000).

[9] Floer, A. Monopoles on asymptotically flat manifolds In: The Floer Memorial Volume, Birkhauser 3-41 (1995).

[10] Floer, A. An instanton invariant for 3-manifolds Commun. Math. Phys. 118 215-240 (1989).

[11] Gromov, M. Pseudoholomorphic curves in symplectic geometry Inventiones Math. 82 307-347 (1985).

[12] Harvey, R. and Lawson, H. B. Calibrated geometries Acta Math. 148 47-157 (1982).

[13] Haydys, A. Generalised Seiberg-Witten equations and hyperkahler geometry Thesis, Gottingen (2006).

[14] Haydys, A. Nonlinear Dirac operators and quaternionic analysis Commun. Math. Phys. 281 251-286 (2008).

[15] Haydys, A. Gauge theory, calibrated geometry and harmonic spinors arxiv 0902.3738.

[16] Hong Y.-J., Harmonic maps into the moduli spaces of flat connections Ann. Global Analysis and Geometry 17 441-473 (1999).

[17] Hohloch, S. Noetal, G. and Salamon, D. Hypercontact structures and Floer homology Geometry and Topology 13 2543-2617 (2009).

[18] Jaffe, A. and Taubes, C.H. Vortices and monopoles Birkhauser, Boston.

[19] Joyce, D. Riemannian holonomy groups and calibrated geometry In: Calabi-Yau manifolds and related geometries 1-68, Universitext, Springer (2003).

[20] Joyce, D. On counting special Lagrangian homology spheres In:Topology and Geometry:Commemorating SISTAG Berrick et al Eds. Contemporary Meth. 314 125-151 Amer. Math. Soc. (1999).

[21] Kovalev, A.G. Twisted connected sums and special Riemannian holonomy J. Reine Angew. Math. 565 125-160 (2003).

[22] Kovalev, A.G. and Nordström, J. Asymptotically cylindrical 7-manifolds with holonomy $G_{2}$, with applications to compact irreducible $G_{2}$-manifolds Annals Global Analysis and Geom. 38 221-257 (2010). 
[23] Leung, N.C. Topological Quantum Field Theory for Calabi-Yau 3-folds and $G_{2}$-manifolds Adv. Theor. Math. Phys. 6 575-591 (2002).

[24] Lewis, C. Spin(7) instantons Oxford University D. Phil thesis (1998).

[25] Maulik, D. , Nekrasov, N. Okounkov, A. and Pandharipande, R. Gromov-Witten theory and Donaldson-Thomas theory, I Compos. Math 142 1263-1285 (2006).

[26] Mclean, R. Deformations of calibrated submanifolds Commun. Analysis and Geometry 6 707-745 (1998).

[27] Oancea, A. and Salamon, D. Lefschetz fibrations, intersection numbers and representations of the framed braid group Preprint arxiv 0708.2051.

[28] Pidstrigatch, V. Hyperkahler manifolds and the Seiberg-Witten equations (English trans.) Proc Steklov Inst. Math. 246 249-262 (2004).

[29] Sá Earp, H. N. Instantons on $G_{2}$-manifolds Imperial College, London Ph. D Thesis (2009).

[30] Salamon, S. M. Riemannian geometry and holonomy groups Pitman Rs. Notes Math. 201 (1989).

[31] Salur,S. Deformations of asymptotically cylindrical coassociative submanifolds with moving boundary DG/0601420.

[32] Tao, T and Tian, G. A singularity removal theorem for Yang-Mills fields in higher dimensions J. Amer. Math. Soc. 17 557-593 (2004).

[33] Taubes, C.H. Stability in Yang-Mills theories Commun. Math. Phys. 91 235-263 (1983).

[34] Taubes, C.H. SW $\Rightarrow$ Gr: from the Seiberg-Witten equation to pseudoholomorphic curves Jour. Amer. Math. Soc. 9 845-918 (1996).

[35] Taubes, C. H. Nonlinear generalisations of a 3-manifold's Dirac operator In: Trends in Math. Phys. AMS/IP Studies Adv. Math. 13 475-486 (1998).

[36] Thomas, R.P. A holomorphic Casson invariant for Calabi-Yau 3-folds and bundles on K3 fibrations Jour. Differential Geometry 54 367-438 (2000).

[37] Thomas, R.P. Moment maps, monodromy and mirror manifolds In Symplectic geometry and mirror symmetry, Seoul 2000 World Scientific 467-498 (2001).

[38] Tian, G. Gauge theory and calibrated geometry Ann. Math. 151 193-268 (2000).

IMPERIAL COLLEGE LONDON

E-mail address: edward.segal04@imperial.ac.uk 
